\title{
The foundation of the theory of the universe dark energy and its nature
}

\author{
Murad Shibli
}

Mechanical Engineering Department, College of Engineering, United Arab Emirates University, Al-Ain, UAE; $\underline{\text { malshibli@,uaeu.ac.ae }}$

Received 1 December 2010; revised 5 January 2011; accepted 8 January 2011.

\section{ABSTRACT}

Surprisingly recent astronomical observations have provided strong evidence that our universe is not only expanding, but also is expanding at an accelerating rate. This paper presents a basis of the theory of universe spacetime dark energy, a solution of Einstein's cosmological constant problem, physical interpretation of universe dark energy and Einstein's cosmological constant Lambda and its value ( = $0.29447 \times 10^{-52} \mathrm{~m}^{-2}$ ), values of universe dark energy density $1.2622 \times 10^{-26} \mathrm{~kg} / \mathrm{m}^{3}=6.8023 \mathrm{GeV}$, universe critical density $1.8069 \times 10^{-26} \mathrm{~kg} / \mathrm{m}^{3}=$ $9.7378 \mathrm{GeV}$, universe matter density $0.54207 \times$ $10^{-26} \mathrm{~kg} / \mathrm{m}^{3}=2.9213 \mathrm{GeV}$, and universe radiation density $2.7103 \times 10^{-31} \mathrm{~kg} / \mathrm{m} 3=1.455 \mathrm{MeV}$. The interpretation in this paper is based on geometric modeling of space-time as a perfect fourdimensional continuum cosmic fluid and the momentum generated by the time. In this modeling time is considered as a mechanical variable along with other variables and treated on an equal footing. In such a modeling, time is considered to have a mechanical nature so that the momentum associated with it is equal to the negative of the universe total energy. Since the momentum associated with the time as a mechanical variable is equal to the negative system total energy, the coupling in the time and its momentum leads to maximum increase in the space-time field with $70.7 \%$ of the total energy. Moreover, a null paraboloid is obtained and interpreted as a function of the momentum generated by time. This paper presents also an interpretation of space-time tri-dipoles, gravity field waves, and gravity carriers (the gravitons). This model suggests that the space-time has a polarity and is composed of dipoles which are responsible for forming the orbits and storing the space-time energy-momentum. The tri-dipoles can be unified into a solo space-time dipole with an angle of $\mathbf{4 5}$ degrees. Such a result shows that the space-time is not void, on the contrary, it is full of conserved and dynamic energy-momentum structure. Furthermore, the gravity field waves is modeled and assumed to be carried by the gravitons which move in the speed of light. The equivalent mass of the graviton (rest mass) is found to be equal to 0.707 of the equivalent mass of the light photons. Such a result indicates that the lightest particle (up to the author's knowledge) in the nature is the graviton and has an equivalent mass equals to $2.5119 \times 10^{-52} \mathrm{~kg}$. Based on the fluidic nature of dark energy, a fourth law of thermodynamics is proposed and a new physical interpretation of Kepler's Laws are presented. Additionally, based on the fact that what we are observing is just the history of our universe, on the Big Bang Theory, Einstein's General Relativity, Hubble Parameter, cosmic inflation theory and on NASA's observation of supernova $1 \mathrm{a}$, then a second-order (parabolic) parametric model is obtained in this proposed paper to describe the accelerated expansion of the universe. This model shows that the universe is approaching the universe cosmic horizon line and will pass through a critical point that will influence significantly its fate. Considering the breaking symmetry model and the variational principle of mechanics, then the universe will witness an infinitesimally stationary state and a symmetry breaking. As result of that, our universe will experience in the near future, a very massive impulse force in the order $10^{83} \mathrm{~N}$. Subsequently, the universe will collapse. Finally, simulation results are demonstrated to verify the analytical results.

Keywords: Dark Energy; Nature of Dark Energy; 
Expansion of the Universe; Einstein's Cosmological Constant; Universe Mass/Energy Densities; Space-Time Dipoles; Gravitons; Fourth Law of Thermodynamics; Fate of the Universe; Kelper's Laws

\section{INTRODUCTION}

Recent astronomical observations by the Supernova Cosmology Project, the High-z Supernova Search Team and cosmic microwave background (CMB) have provided strong evidence that our universe is not only expanding, but also expanding at an accelerating rate [1-8]. It was only in 1998 when dark energy proposed for the first time, after two groups of astronomers made a survey of exploding stars, or supernovas Ia, in a number of distant galaxies $[1,3]$. These researchers found that the supernovas were dimmer than they should have been, and that meant they were farther away than they should have been. The only way for that to happen, the astronomers realized, was if the expansion of the universe had sped up at some time in the past, as well as accounting for a significant portion of a missing component in the universe [9]. The only explanation is that there is a kind of force that has a strong negative pressure and acting outward in opposition to gravitational force at large scales which was proposed for the first time by Einstein in his General Relativity and given the name the cosmological constant Lambda [10]. This force is given the name Dark Energy, since it is transparent and can not be observed or detected directly. The fourth law of thermodynamics is proposed by the author to account for the dark energy [11].

Moreover, in Nov. 2006 Scientists using NASA's Hubble Telescope have discovered that the universe has been expanding as long as nine billion years ago. Members of High-z Supernova Team and Supernova Cosmology Project used Hubble to detect the acceleration of the expansion of the space from observations of distant supernovae aged between three to ten billion years. The objective was to uncover two of dark energy's most fundamentals properties: its strength and its permanence. This paper will be published by NASA's group in March 2007. Before that, it is thought that the expansion was decelerating, due to the attractive influence of dark matter and baryons. The density of dark matter in an expanding universe disappears more quickly than dark energy, and eventually the dark energy dominates. If the acceleration continues indefinitely, the ultimate result will be that galaxies outside the local supercluster will move beyond the cosmic horizon: they will no longer be visible, because their line-of-sight velocity becomes greater than the speed of light.

These cosmological observations strongly suggest that the universe is dominated by a smoothly homogenous distributed dark energy component [9,12-21]. The quantity and composition of matter and energy in the universe is a fundamental and important issue in cosmology and physics. Based on the Lambda-Cold Dark Matter Model (Lambda-CDM 2006), dark energy contributes about $70 \%$ of the critical density and has a negative pressure. The cold dark matter contributes $25 \%$, Hydrogen, Helium and stars contributes $5 \%$ and, finally the radiation contributes $5 \times 10^{-5}$. The measurements of the Wilkinson Microwave Anisotropy Probe (WMAP) satellite indicate the universe geometry is very close to flat $[22,23]$.

The dark energy that is causing the accelerated expansion of the universe is still poorly understood. What is known so far is that it contributes about $70 \%$ of the critical density and has a negative pressure $[9,19,20]$. According to the theory of General Relativity, the effect of such a negative pressure is qualitatively similar to a force acting in opposition to gravity at large scales. Such astronomical observations have raised fundamental issues: 1) what is the nature of smoothly-distributed energy which apparently dominates the universe $(70 \%), 2)$ why the vacuum energy is much smaller than the theory can predict? 3) why dark energy density is approximately equal to the matter density today? A Physical insight of dark energy and how to detect it using micro-nano space robotic system was proposed in [24-26].

Modern theoretical scientific exploration of the ultimate fate of the universe became possible with Albert Einstein's 1915 theory of general relativity. Alexander Friedmann proposed one such solution in 1921. This solution implies that the universe has been expanding from an initial singularity; this is, essentially, the Big Bang.

According to the Big Bang Model, inflation suggests that there was a period of exponential expansion in the very early universe probably lasted roughly $10^{-33}$ seconds [27]. During inflation, the universe undergoes exponential expansion so that large regions of space are pushed beyond our observable horizon. After inflation, the universe expands according to Hubble's law, and regions that were out of casual contacts come back into the horizon. During inflation, the universe is flattened and the universe enters a homogeneous and isentropic rapidly expanding phase is which seeds of structure formation are laid down in form of a primordial spectrum of nearlyscale-invariant fluctuations. This explains the observed isotropy of CMB.

The cosmic inflation may occur during the grand unification symmetry is broken. Symmetry breaking in physics describes a phenomenon where infinitesimally fluctuations acting on the universe crossing a critical point decide its fate by determining which branch of a bifurcation is taken. This process is called symmetry 
breaking because such transitions usually bring the universe from a disorderedly state into one that is more order or vice versa.

Based on the measurements of the expansion of the universe using type 1a supernovae, measurements of the cosmic microwave background, the measurements of the correlation function of galaxies, the universe has a calculated age of $13.7+/-0.2$ billion years. Such a result is presented in the lambda-Cold Dark Matter model (Lambda-CDM) which is a mathematical model of the Big Bang with six free parameters.

The discovery of cosmic microwave background radiation (CMB) by Arno Penzias and Robert Wilson in 1965 was a straightforward prediction of the Big Bang theory. The Big Bang immediately became the most widely held view of the origin of the universe. Based on the Big bang theory, the Cosmic Microwave Background (CMB) receiving the photons just 300,000 years after the Big Bang, Lambda-CDM [22,23] which estimates that the age of the universe by 13.7 billion years, the Einstein's General theory that both space and time are one entity and are not separated, and on Friedman proposed solution of Einstein's General theory which implies that the universe has been expanding from an initial singularity; this is, essentially, the Big Bang, based on all of that it can be concluded that the universe which is structured and composed of homogeneous conserved energy-mass space-time structure. Then, before the Big Bang it was null and then it was created and has been expanding since then. Accordingly, the space and time has a beginning signed by the Big Bang.

There are some very speculative ideas about the future of the universe. One suggests that phantom energy causes divergent expansion, which would imply that the effective force of dark energy continues growing until it dominates all other forces in the universe. Under this scenario, dark energy would ultimately tear apart all gravitationally bound structures, including galaxies and solar systems, and eventually overcome the electrical and nuclear forces to tear apart atoms themselves, ending the universe in a Big Rip [27]. On the other hand, dark energy might dissipate with time, or even become attractive. Such uncertainties leave open the possibility that gravity might yet rule one day and lead to a universe that contracts in on itself in a "Big Crunch". Some scenarios, such as the cyclic model suggest this could be the case.

An important parameter in fate of the universe theory is the density parameter, Omega $(\Omega)$, defined as the average matter density of the universe divided by a critical value of that density [24-26]. This creates three possible ultimate fates of the universe, depending on whether $\Omega$ is equal to, less than, or greater than 1 . These are called, respectively, the Flat, Open and Closed universes. These three adjectives refer to the overall geometry of the universe, and not to the local curving of spacetime caused by smaller clumps of mass (for example, galaxies and stars). Astronomers using the Hubble Space Telescope in 2004 unveiled the deepest look into the universe yet, a portrait of what could be the most distant galaxies ever seen just 300-800 million years after the big bang from. This image is called the Hubble Ultra Deep Field (HUDF). Furthermore, rec universe's first trillionth of a second.ent results from the WMAP probe have allowed scientists to see what is thought.

This paper presents a basis of the theory of universe space-time dark energy, a solution of Einstein's cosmological constant problem, physical interpretation of universe dark energy and Einstein's cosmological constant Lambda and its value $\left(=0.29447 \times 10^{-52} \mathrm{~m}^{-2}\right)$, values of universe dark energy density $\left(=1.2622 \times 10^{-26} \mathrm{~kg} / \mathrm{m}^{3}=\right.$ $6.8023 \mathrm{GeV})$, critical density $\left(1.8069 \times 10^{-26} \mathrm{~kg} / \mathrm{m}^{3}=\right.$ $9.7378 \mathrm{GeV})$, matter density $\left(=0.54207 \times 10^{-26} \mathrm{~kg} / \mathrm{m}^{3}=\right.$ $2.9213 \mathrm{GeV})$, and universe radiation density $(=2.7103 \times$ $\left.10^{-31} \mathrm{~kg} / \mathrm{m}^{3}=1.4558 \mathrm{MeV}\right)$. The interpretation in this paper is based on geometric modeling of space-time as a perfect four-dimensional continuum cosmic fluid and the momentum generated by the time. In this modeling time is considered as a mechanical variable along with other variables and treated on an equal footing. In such a modeling, time is considered to have a mechanical nature so that the momentum associated with it is equal to the negative of the universe total energy. It is found that dark energy is a property of the space-time itself.

Since the momentum associated with the time as a mechanical variable is equal to the negative system total energy, the coupling in the time and its momentum leads to maximum increase in the space-time field. The amount of energy which contributes to this increase is found to be $70.7 \%$ of the total energy. Moreover, a null paraboloid is obtained and interpreted as a function of the momentum generated by time. This paper presents also an interpretation of space-time tri-dipoles, gravity field waves, and gravity carriers (the gravitons). This model suggests that the space-time has a polarity and is composed of dipoles which are responsible for forming the orbits and storing the space-time energy-momentum. The tri-dipoles can be unified into a solo space-time dipole with an angle of 45 degrees. Such a result shows that the space-time is not void, on the contrary, it is full of conserved and dynamic energy-momentum structure. Furthermore, the gravity field waves is modeled and assumed to be carried by the gravitons which move in the speed of light. The equivalent mass of the graviton (rest mass) is found to be equal to 0.707 of the equivalent mass of the light carrier (the photon). Such a result indicates that the lightest particle (up to the author's knowledge) in the nature is the gra- 
viton and has an equivalent mass equals to $2.5119 \times 10^{-52}$ $\mathrm{kg}$. Moreover, based on the fluidic nature of dark energy, the fourth law of thermodynamics is proposed, a new formulation and physical interpretation of Kepler's Three Laws are presented.

Additionally, based on the fact that what we are observing is just the history of our universe, on the Big Bang Theory, Einstein's General Relativity, Hubble Parameter, the estimated age of the universe, cosmic inflation theory and on NASA's observation of supernova 1a, then a second-order (parabolic) parametric model is obtained in this proposed paper to describe the accelerated expansion of the universe. This model shows that the universe is approaching the universe cosmic horizon line and will pass through a critical point that will influence significantly its fate. Considering the breaking symmetry model and the variational principle of mechanics, then the universe will witness an infinitesimally stationary state and a symmetry breaking. Considering the breaking symmetry model and the variational principle of mechanics, then the universe will witness an infinitesimally stationary state and a symmetry breaking. As result of that, our universe will experience in the near future (relative to the age of the universe) a very massive impulse force in the order $10^{83}$ N. Finally, simulation results are demonstrated to verify the analytical results.

This proposed paper is organized as follows. In Section 2 modeling of the space-time universe is presented, Section 3 describes the modeling of gravity waves and gravitons. Section 4 proposes space-time dipoles. Seven independent proofs of the nature of dark energy and its nature, the fourth law of thermodynamics are presented in Section 5. Section 6 explains why the universe is expanding and calculates Einstein's cosmological constant and the value of dark energy density and other mass/ energy densities. Section 7 presents a new formulation of Kepler's laws. The parametric model of the ultimate fate of universe is suggested in Section 8. Simulation re- sults of the analytical models are demonstrated in 9. Finally, the paper is concluded by Section 10 .

\section{MODELING OF THE SPACE-TIME UNIVERSE}

Great advantage occurs sometimes through letting the time $t$ become one of the mechanical variables. Instead of considering the generalized position coordinates $\theta_{i}$ as a function of the time $t$, we consider the position coordinates $q_{i}$ and the time $t$ as mechanical variables, giving them as a function of some unspecified parameter $\varsigma$. In relativistic mechanics this procedure is an absolute necessity since space and time are united into four-dimensional world of Einstein and Minkovski. Hence the Lagarngian configuration space has $N+1$ dimen- sions, we now add the time $t$ to the generalized coordinates $q_{i}$ as

$$
t=q_{N+1}
$$

Then, the corresponding phase space must comprise of $2 N+2$ corresponding to the $N+1$ pairs of canonical variables

$$
\left(\begin{array}{l}
q_{1} \\
p_{1}
\end{array}\right),\left(\begin{array}{l}
q_{2} \\
p_{2}
\end{array}\right), \ldots,\left(\begin{array}{l}
q_{N} \\
p_{N}
\end{array}\right),\left(\begin{array}{l}
q_{N+1} \\
p_{N+1}
\end{array}\right)
$$

The system now has $N+1$ degrees of freedom. Denoting derivative with respect to $\varsigma$ by a prime "' ", the system action integral appears in the form

$$
' W=\int_{\varsigma_{1}}^{\varsigma_{2}} L\left(q_{1}, q_{2}, \cdots, q_{N} ; \frac{q_{1}^{\prime}}{t^{\prime}}, \frac{q_{2}^{\prime}}{t^{\prime}}, \cdots, \frac{q_{N}^{\prime}}{t^{\prime}}\right) t^{\prime} d \varsigma
$$

For this purpose let the momentum associated with the time $t$ denoted as $p_{t} \equiv p_{N+1}$ be formed as

$$
\begin{aligned}
p_{t}=\frac{\partial\left(L t^{\prime}\right)}{\partial t^{\prime}} & =L-\left(\sum_{i=1}^{N} \frac{\partial L}{\partial \dot{q}_{i}} \frac{q_{i}^{\prime}}{t^{\prime 2}}\right) t^{\prime} \\
p_{t} & =L-\sum_{i=1}^{N} p_{i} \dot{q}_{i} \\
p_{t} & =-\left(\sum_{i=1}^{N} p_{i} \dot{q}_{i}-L\right)
\end{aligned}
$$

The last Eq.6 is exactly the negative of the total energy of the system. This leads to the conclusion that the momentum associated with the time is the negative of the system total energy [6]. That is, every system is conserved when the time is considered a mechanical variable. In other words

$$
p_{t}=-E_{\text {TOTAL }}
$$

Now the Hamiltonian function can be extended by adding the time as a mechanical variable as

$$
\Lambda_{E X T}=K\left(q_{1}, q_{2}, \cdots, q_{N+1} ; p_{1}, p_{2}, \cdots, p_{N+1}\right)=\text { const }
$$

In the absence of external forces that the conservation of momentum of a mechanical system can be extended to

$$
P=\sum_{i=1}^{N+1} p_{i}=p_{1}+p_{2}+\cdots+p_{N+1}=0
$$

or

$$
\sum_{i=1}^{N} p_{i}=p_{1}+p_{2}+\cdots+p_{N}=-p_{N+1}=E_{T O T}
$$

The last Eq.10 indicates that total momentum of the system is conserved but with a drift. This drift is due to considering the time as a mechanical variable. As mentioned before in this section the objective to describe the gravitational field generated by the rotating of the mother 
spacecraft with high relative mass comparing to the micro spacecraft.

The line-element in this field can be described in terms of four variables: three for the space $q_{1}, q_{2}, q_{3}$ and one for the time $q_{4}$. The surface of the gravitational field can be given as

$$
f\left(q_{1}, q_{2}, q_{3}, q_{4}\right)=q_{1}^{2}+q_{2}^{2}+q_{3}^{2}+f_{t}\left(q_{4}\right)-R^{2}=0
$$

where $f_{t}\left(q_{4}\right)$ is a function of the variable time and will be determined later, and $R$ is the radius of the sphere. The motion of the micro spacecraft robot is restricted to this surface.

Now let us introduce the line element

$$
d s=F\left(q_{1}, q_{2}, q_{3}, q_{4} ; d q_{1}, d q_{2}, d q_{3}, d q_{4}\right)
$$

where the function $F$ is an arbitrary function of eight variables, except for the restriction that to is assumed to be homogeneous differential form of the first order in term of the displacements $d q_{i}$. This means that

$$
\begin{aligned}
d s & =F\left(q_{1}, q_{2}, q_{3}, q_{4} ; a d q_{1}, a d q_{2}, a d q_{3}, a d q_{4}\right) \\
& =F\left(q_{1}, q_{2}, q_{3}, q_{4} ; d q_{1}, d q_{2}, d q_{3}, d q_{4}\right)
\end{aligned}
$$

An arbitrary curve of this manifold can be given in a parametric form as follows

$$
q_{i}=f_{i}(\varsigma)
$$

where $\varsigma$ is an arbitrary parameter. The line-element $d s$ can be modified to

$$
d s=F\left(q_{1}, q_{2}, q_{3}, q_{4} ; q_{1}^{\prime}, q_{2}^{\prime}, q_{3}^{\prime}, q_{4}^{\prime}\right) d \varsigma
$$

Hence the problem of minimizing the length of a curve between two points $\varsigma_{1}$ and $\varsigma_{2}$, leads to the minimizing the volitional integral

$$
I=\int_{\varsigma_{1}}^{\varsigma_{2}} F\left(q_{1}, q_{2}, q_{3}, q_{4} ; q_{1}^{\prime}, q_{2}^{\prime}, q_{3}^{\prime}, q_{4}^{\prime}\right) d \varsigma
$$

Let the momenta $p_{i}$ be defined as

$$
p_{i}=\frac{\partial F}{\partial q_{i}^{\prime}}
$$

Taking into consideration that the function $F$ is homogeneous function of first order in terms of the variables $q_{i}^{\prime}$, then the Hamiltonian function $H$ can be constructed as

$$
H=\sum_{i=1}^{4} \frac{\partial F}{\partial q_{i}^{\prime}} q_{i}^{\prime}-F=0
$$

along with the identity

$$
K\left(q_{1}, q_{2}, q_{3}, q_{4} ; p_{1}, p_{2}, p_{3}, p_{4}\right)=0
$$

Now going back to the surface manifold to wish the micro spacecraft robot is restricted. Let us assume that two points come arbitrary near to each other such that

$$
q_{i}=\bar{q}_{i}+d q_{i}
$$

It is interesting to find the shortest distance of the point $\bar{q}_{i}$ from an arbitrary point on the surface. Then the minimization of a function $W\left(q_{i}, \bar{q}_{i}\right)$ subjected to the constraint (11) where

$$
\begin{aligned}
& W\left(q_{i}, \bar{q}_{i}\right) \\
& =\left(q_{1}, q_{2}, q_{3}, q_{4}, q_{1}-d q_{1}, q_{2}-d q_{2}, q_{3}-d q_{3}, q_{4}-d q_{4}\right)
\end{aligned}
$$

Now the minimization problem can be given by

$$
\frac{\partial W}{\partial q_{i}}-\lambda \frac{\partial f}{\partial q_{i}}=0
$$

Making a benefit of the properties of Hamilton's principle function then the momenta is

$$
p_{i}=\lambda \frac{\partial f}{\partial q_{i}}
$$

This equation represents the direction cosines of the normal in terms of the gradient of $f$.

It is possible to rewrite (18) in the form

$$
\sum p_{i} d q_{i}=d s
$$

Substituting (23) into (24) yields

$$
d s=\lambda \sum \frac{\partial f}{\partial q_{i}} d q_{i}=\lambda d f
$$

From (25),

$$
\frac{d f}{d s}=\frac{1}{\lambda}=|\operatorname{grad} f|
$$

where $1 / \lambda$ is the maximum rate of change of the function $f$ at that given point along the normal (because it is known from vector calculus that the directional derivative characterizes the maximum increase in function $f$ ).

Considering (26) then (23) can be written as

$$
p_{i}=\frac{1}{|\operatorname{grad} f|} \frac{\partial f}{\partial q_{i}}
$$

According to the theorem that the momentum associated with the time is the negative of the system total energy, then (27) becomes

$$
\frac{\partial f}{\partial q_{4}}=-E_{\text {TOTAL }}|\operatorname{grad} f|
$$

Note that if the gradient value is other than zero it characterizes the maximum increase, then $|\operatorname{grad} f|>0$.

For example for a spherical field, the maximum rate of change (directional derivative) is 1 because the gradient is the normal to the sphere surface and taken along the radius, in this case (28) becomes 


$$
p_{4}=\frac{\partial f}{\partial q_{4}}=-E_{\text {TOTAL }}
$$

Using (29) the function $f_{t}\left(q_{4}\right)$ can now be determined as

$$
f_{t}\left(q_{4}\right)=-E_{\text {TOTAL }} q_{4}
$$

By this the surface of the sphere can be represented as

$$
q_{1}^{2}+q_{2}^{2}+q_{3}^{2}-E_{\text {TOTAL }} q_{4}-R^{2}=0
$$

As a general case, the gravitational surface should be modified to

$$
q_{1}^{2}+q_{2}^{2}+q_{3}^{2}-|\operatorname{grad} f| E_{\text {TOTAL }} q_{4}-R^{2}=0
$$

Eq.32 has a physical interpretation, specifically the term $\operatorname{grad} f \mid E_{\text {TOTAL }} q_{4}$, it shows that time as a mechanical variable along with its associated momentum which is equal to the negative of the system total energy play a significant role in determining the size of the gravitational field under consideration and characterized by the maximum increase $|\operatorname{grad} f|$.

Recalling the kinetic energy of a unit mass which can be given in the form

$$
T=\frac{1}{2}\left(\frac{d s}{d t}\right)^{2}
$$

where $d s$ is the line element of the configuration space. Since now the time $t$ is no longer an independent variable but $\varsigma$, the kinetic energy (33) should be modified to

$$
T=\frac{1}{2}\left(\frac{d s}{d \varsigma}\right)^{2} / t^{\prime 2}
$$

or

$$
\left(\frac{d s}{d \varsigma}\right)^{2}=2 T \cdot t^{\prime 2}
$$

From (35) it can be seen that

$$
d s \propto \sqrt{2}
$$

Using (36) and (26) it yields

$$
|\operatorname{grad} f| \propto \frac{1}{\sqrt{2}}=0.707
$$

Making a benefit of (37) and substituting this value in (28) and (32), respectively, one gets

$$
\begin{gathered}
\frac{\partial f}{\partial q_{4}}=-0.707 E_{\text {TOTAL }} \\
q_{1}^{2}+q_{2}^{2}+q_{3}^{2}-0.707 E_{\text {TOTAL }} q_{4}-R^{2}=0
\end{gathered}
$$

The physical significance of (38) and (39) is that the amount of energy that contributes to the maximum increase is proportional to the total energy by 0.707 . This energy is proposed as the universe dark energy which is behind the expansion. The value 0.707 ( $=70.7 \%$ of total energy) agrees with the measured values reported recently in $[1-4,10]$. It is found for a flat universe the dark energy $=0.71$ in [15]. Eq.39 can be modified to

$$
q_{1}^{2}+q_{2}^{2}+q_{3}^{2}=0.707 E_{\text {TOTAL }} q_{4}+R^{2}
$$

For a given conserved mechanical system, the right hand side of (40) shows that the surface that describes the space-time field is expanding. This expansion is due to the coupling in time and its momentum and characterized by the maximum increase $|\operatorname{gard} f|=0.707$. The more the system has energy the more it is expanding. That explains why galaxies and clusters are departing much faster that our solar system. For a conserved mechanical system, the line-element of this manifold now can be given in the form

$$
d s^{2}=d q_{1}^{2}+d q_{2}^{2}+d q_{3}^{2}-0.707 E_{\text {TOTAL }} d q_{4}
$$

To compare this manifold with Robertson-Walker and Minkowski's four-dimensional world are defined as follows. For a flat universe represented in Robertson-Walker cosmology defined as

$$
d s^{2}=a(t)\left(d x^{2}+d y^{2}+d z^{2}\right)-c^{2} d t^{2}
$$

where $c$ is the speed of light and $a(t)$ is the expansion rate. Meanwhile, for a Minkowski's four-dimen- sional world it is defined as

$$
d s^{2}=d q_{1}^{2}+d q_{2}^{2}+d q_{3}^{2}-c^{2} d q_{4}^{2}
$$

The difference occurs in the coefficient of the time displacement related terms so that in (42) and (43) it is equal to the $\left(-c^{2} d q_{4}^{2}\right)$, meanwhile in the proposed model (41) it is equal to $\left(-0.707 E_{\text {TOTAL }} d q_{4}\right)$. The previous analysis can be summarized in the following theorem.

Theorem 1: Considering the time as a dependent mechanical variable along with other generalized variables, the gravitational field described in (39) is expanding due to the coupling in time and its momentum (34) and characterized by the maximum increase $|\operatorname{gard} f|=0.707$.

Three cases can be also concluded from (43)

Case 1: if $d s^{2}<0$, then

$$
0.707 E_{\text {TOTAL }} d q_{4}>\left(d q_{1}^{2}+d q_{2}^{2}+d q_{3}^{2}\right)
$$

and the curve is time-like in that interval.

Case 2: if $d s^{2}>0$, then

$$
0.707 E_{\text {TOTAL }} d q_{4}<\left(d q_{1}^{2}+d q_{2}^{2}+d q_{3}^{2}\right)
$$

and the curve is space-like in that interval.

Case 3: if $d s^{2}=0$, then

$$
0.707 E_{\text {TOTAL }} d q_{4}=d q_{1}^{2}+d q_{2}^{2}+d q_{3}^{2}
$$

and the curve is null in that interval. The reduced line- 
element (46) represent a null paraboloid which depends on the system total energy $E_{\text {TOTAL }}$ and the maximum increase. In this case if a particle is moving with the speed of light then the null rays will lie on the surface of the paraboloid.

\section{MODELING OF GRAVITY WAVES AND GRAVITONS}

In the following analysis we assume that we are dealing with the motion of a particle in the gravitational field of a total energy $E$ and a potential energy $V$. Based on the previous analysis and the line-element, we consider now a gravitational field function $S=S\left(q_{1}, q_{2}, q_{3}, q_{4}\right)$, a function of 3 space coordinates $q_{1}, q_{2}, q_{3}$ and a time coordinate $q_{4}$, treated on an equal footing and satisfies the following wave equation follows

$$
\left(\frac{\partial S}{\partial q_{1}}\right)^{2}+\left(\frac{\partial S}{\partial q_{2}}\right)^{2}+\left(\frac{\partial S}{\partial q_{3}}\right)^{2}+\left(\frac{\partial S}{\partial q_{4}}\right)^{2}=2 m(E-V)
$$

where $m$ is the equivalent mass of the particle that carries the gravitational waves and will be calculated in the following analysis. It is known now from the previous analysis that the gradient of the gradient of a surface function $S$ has the maximum increase and has the direction of the normal to the surface

$$
S=S\left(q_{1}, q_{2}, q_{3}, q_{4}\right)=\text { const. }
$$

As derived in the previous section, the directional derivative (the absolute value of $S$ ) can be defined as

$$
|\operatorname{gard} S|=\frac{\partial S}{\partial q}
$$

and taken in the direction of the normal. Referring to Figure 1, it possible to write (49) in the form

$$
|\operatorname{gard} S|=\frac{\varepsilon}{\delta}
$$

The gradient (47) can be rewritten as follows

$$
\frac{\varepsilon}{\delta}=\sqrt{2 m(E-V)}
$$

The gradient (49) represents the linear momentum such that

$$
|\operatorname{gard} S|=\frac{\partial S}{\partial q}=m v
$$

Based on (41) the maximum increase is found to be 0.707 , then (51) and (52) yield to:

$$
\frac{\partial S}{\partial q}=m v=0.707
$$

Since the Einstein's Relativity assumes that the maximum speed in the nature is the speed of light, and

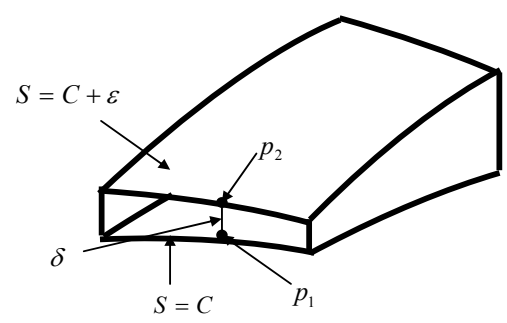

Figure 1. Gravitational wave construction.

assuming that the gravitational waves moves in the speed of light

$$
v=\text { speed of light }=c
$$

and assuming that the speed of light is considered as a unity and from (53) we conclude that the mass of the gravitons which carries the gravity waves is defined as

$$
m_{\text {graviton }}=0.707 m_{\text {photon }}
$$

This indicates that the lightest particle in the nature is the graviton and has an equivalent mass equals to 2.5119 $\times 10^{-52} \mathrm{~kg}$ (the equivalent mass of the photon is $3.9 \times$ $10^{-22}$ of the mass of the electron). That is, the electron is heavier than the graviton by $3.6267 \times 10^{21}$ times.

Theorem 2: (Gravity Waves and Gravitons): The gravity waves described by (47) is carried by the gravitons which move in the speed of light and the graviton has an equivalent mass equals to $2.5119 \times 10^{-52} \mathrm{~kg}$.

\section{MODELING OF SPACE-TIME DIPOLES}

The Euler-Lagrangian equation takes the following form in case of a 4-Dimnesional space-time world, which depends on the coordinates: $x=q_{1}, y=q_{2}, z=q_{3}$, $t=q_{4}$,

$$
\operatorname{div} \boldsymbol{P}_{\text {Total }}=\frac{\partial p_{1}}{\partial q_{1}}+\frac{\partial p_{2}}{\partial q_{2}}+\frac{\partial p_{3}}{\partial q_{3}}+\frac{\partial p_{4}}{\partial q_{4}}=0
$$

When dealing with a particle, only $t=q_{4}$ is considered, and (56) is reduced to

$$
\frac{\partial p_{4}}{\partial q_{4}}=0
$$

Eq.57 implies that the time momentum is constant which by the virtue of Theorem (1) is interpreted as the system total energy, that is

$$
p_{4}=-E_{\text {Total }}=\text { const } \text {. }
$$

Eq.57 suggests that $p_{4}$ is not a function of the time coordinate $t=q_{4}$.

Now by the virtue of Neother's Invariant Theorem [6,8] and Divergence Transformation Theorem of Gauss, (56) can be interpreted as follows: 


$$
\iiint_{V} \operatorname{div} \boldsymbol{P} d V=\iint_{S} \boldsymbol{P} \cdot n d A
$$

where $P$ is the momentum vector of the 3 -domensional space, $n$ is the normal vector to the surface $S$ and $d V$ is the segment of volume $V$. Now by considering that the momentum of time (as a mechanical variable) is constant and equals to the system total energy as in (58), then (59) can be modified as

$$
\iint_{S} \boldsymbol{P} \cdot \boldsymbol{n} d A=-\frac{d}{d q_{4}} \iiint_{V} p_{4} d V
$$

The physical interpretation of (59) and (60) as follows: the space-time total energy-momentum contained in a certain volume is equal to the negative of the total space-time energy-momentum that flows through the boundary surface of that certain volume. In sense of the previous analysis an equivalent equation can be drawn from (56) as:

$$
\frac{\partial p_{4}}{\partial q_{4}}+\operatorname{div} \boldsymbol{P}=0
$$

The importance of (61) is the condition for continuity equation of the space-time.

The world line-element proposed in (41) and Minkovisky world shows that there is a polarity in the spacetime description $(+,+,+,-)$ : a plus $(+)$ is a space signature, meanwhile a minus $(-)$ is a time signature. This polarity can also be suggested by applying the system conservation of momentum with zero initial conditions

$$
p_{1}+p_{2}+p_{3}+p_{4}=0
$$

or by virtue of (21)

$$
p_{1}+p_{2}+p_{3}-E_{\text {Total }}=0
$$

This suggests that space-time exists in a dipole form originated at the time $q_{4}$ and ended at the space resultant vector $q\left(q_{1}, q_{2}, q_{3}\right)$. The space-time dipole moment vector is denoted by $e$. Assuming also that the dipole is placed in a uniform gravity field $\Phi$ and assuming that the dipole makes an angle $\beta$ with the field. This produces a net torque on the dipole expressed as the cross product of the space-time dipole moment and the field:

$$
\tau=e \times \Phi=e \Phi \sin \beta
$$

The potential energy can be expressed as a function of the orientation of the space-time dipole with the gravity field:

$$
U=-e \cdot \Phi=-e \Phi \cos \beta
$$

Eqs.64 and $\mathbf{6 5}$ are another representation of $(62,63)$. Since the time momentum is equal to the negative of system total energy, then from (64) and (65) the orientation angle should be 45 degrees (that is $\sin \beta=$ $\cos \beta=0.707)$. The figure 0.707 has a special signifi- cance in the space-time. The space-time is made up of dipoles. The dipoles are oriented with 45 degrees. When a gravity field is applied, a torque on the dipoles is produced. The space-time dipoles align themselves with the gravity field. The degree of alignment of the space-time dipoles with the field depends on strength of the gravity field and the mass and angular velocity. This insight can be added to Theorem 1 where only 0.707 of the total energy contributes to the expansion of the universe and this energy is given the name Dark Energy.

Theorem 3: (Space-Time Dipoles): the space-time described by (60), (62) and (63) is composed of spacetime dipoles and the dipole moment is oriented by 45 degrees originated from the time and ended at the space and these dipoles are behind the formation of orbits structure.

Theorem 3 is derived based on Noether's Invariant Variational Theorem, Euler-Lagarangian equations and the Divergence Theorem of Gauss. This theorem can also be proved by physical tracking of planetary, solar and galactic orbits as shown in Figures $\mathbf{2}$ and $\mathbf{3}$.

\section{NATURE OF DARK ENERGY}

The purpose of this section to reveal the nature of themysterious dark energy and its governing law that is causing the universe to expand at an accelerating rate. Seven independent proofs are provided.

Proof 1 (Classical Thermodynamics): Since the universe is expanding, then its volume is increasing and its
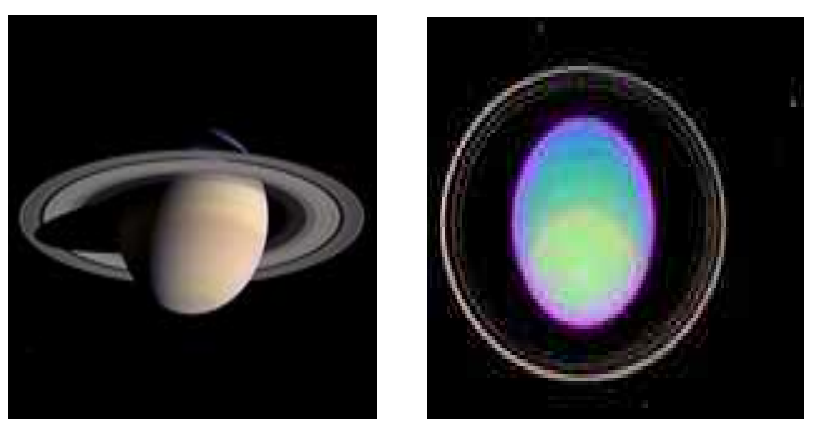

Figure 2. Saturn and its rings, uranus and its rings.
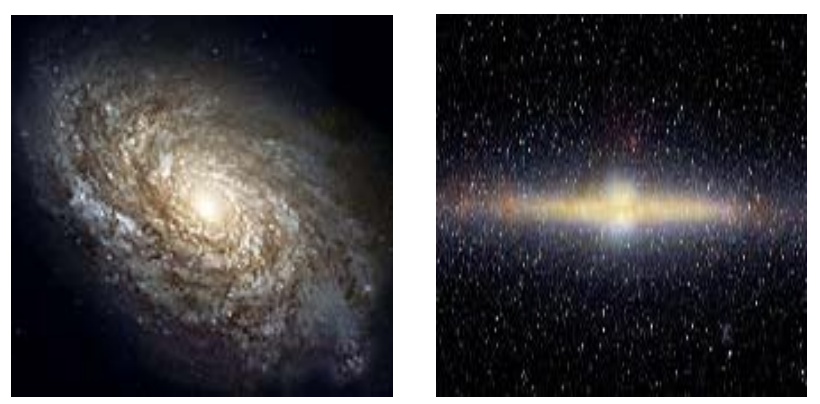

Figure 3. Spiral galaxy and the milky way galaxy. 
boundaries are moving. A closed system composed of homogenous isotropic cosmic fluid (space-time universe), with moving boundaries is then considered in this proof. In classical thermodynamics the expansion work is often called moving boundary work [28].

The objective is now to determine the moving boundary work of expanding universe assuming a quasi-equilibrium expansion process. Consider that the system is experiencing a negative pressure $-\mathrm{P}$, and the pressure force acting on a differential area $d A$ is $-\mathrm{P} d A$ acts outward normal to the surface. Under the influence of the negative pressure the system will move outward a differential distance $d s$ and the system is undergoing a change in its volume equal to $d V=d A d s$, where $V$ is the total volume as shown in Figure 4. Noting that work is force times distance, then the boundary work done on the system is expressed by

$$
\delta W=-F d s=-\mathrm{P} d A d s=-\mathrm{P} d V
$$

Note that both $\delta$ and $d$ are used to indicate differential quantities, but $\delta$ is typically used for quantities that are path functions (such as work) and have inexact differentials, while $d$ is used for quantities that are point functions and have exact differential. Taking the integration of both sides of Eq.66 yields to

$$
W=-\mathrm{P} V
$$

where $V$ is the net final total volume. Defining the amount of energy exists per unit volume as the energy density $\rho$. Then, the amount of dark energy contained inside the universe system undergoing an increase in its volume is

$$
E=\rho V
$$

By the virtue of first law of thermodynamics of energy conservation and by comparing (67) with (68) yields

$$
\mathrm{P}=-\rho
$$

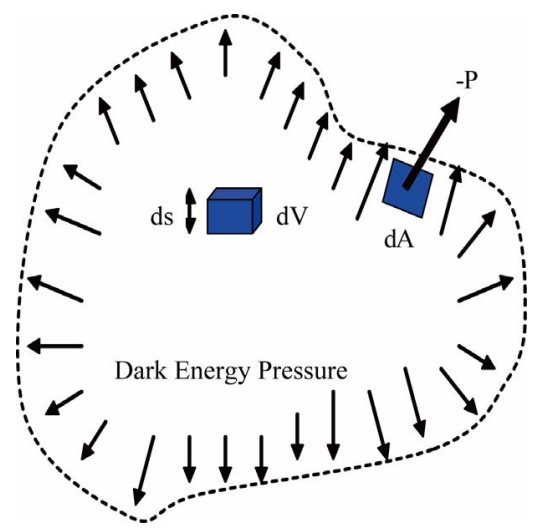

Figure 4. The differential surface of a space-time universe system with arbitrary shape under the influence of expansion by the negative pressure of dark energy.
The physical interpretation of (69) is that the negative pressure that is causing the universe to expand is equal to the negative of energy volumetric density. This force is behind the expansion of the universe.

Proof 2 (The Thermodynamics Equation of State of an Ideal Gas): this equation relates the pressure $P$, temperature $T$ and the volume $V$ of a substance behaves as an ideal gas [28], that is

$$
\mathrm{P} V=m R T=E
$$

Note that both sides of the equation have the units of energy (work done by pressure P). Assume now that dark energy behaves like an ideal gas with a negative pressure -P that causes the universe to expand with a total volume $V$, then by dividing both side of the equation of state (71) by $V$, it becomes

$$
\mathrm{P}=-\frac{m R T}{V}=-\frac{E}{V}=-\rho
$$

It is worth to mention that NASA's Cosmic Microwave Background Explorer (CMB) in 1992 estimated that the sky has a temperature close to 2.7251 Kelvin. Moreover, the Wilkinson Microwave Anisotropy Probe (WMAP) in 2003 has made a map of the temperature fluctuations of the CMB with more accuracy [8].

Proof 3 (The Cosmological Equation of State of a Perfect Fluid): the equation of state of a perfect fluid in cosmology is characterized by a dimensionless number $w$ [9], equal to the ratio of the pressure of the fluid to its energy density as follows

$$
\mathrm{P}=w \rho
$$

Recent observations of Lambda-Cold Dark Matter model (L-CDM) estimate the value of $w$ of a flat universe close to -1 , that is

$$
\mathrm{P}=-\rho
$$

Proof 4 (Invariant Variational Principle of Mechanics): for the importance of this proof some details here will be repeated so that all proofs together can be treated at the same time. Hence the Lagarngian configuration space has $N+1$ dimensions, we now add the time $t$ to the generalized coordinates $\theta_{i}$ as

$$
t=q_{N+1}
$$

Then, the corresponding phase space must comprise of $2 N+2$ corresponding to the $N+1$ pairs of canonical variables:

$$
\left(\begin{array}{l}
q_{1} \\
p_{1}
\end{array}\right),\left(\begin{array}{l}
q_{2} \\
p_{2}
\end{array}\right), \ldots,\left(\begin{array}{l}
q_{N} \\
p_{N}
\end{array}\right),\left(\begin{array}{l}
q_{N+1} \\
p_{N+1}
\end{array}\right)
$$

The system now has $N+1$ degrees of freedom (DOF). Denoting derivative with respect to $\tau$ by a prime "'", 
the system action integral appears in the form

$$
W=\int_{\tau_{1}}^{\tau_{2}} L\left(q_{1}, q_{2}, \cdots, q_{N+1} ; \frac{q_{1}^{\prime}}{t^{\prime}}, \frac{q_{2}^{\prime}}{t^{\prime}}, \cdots, \frac{q_{N+1^{\prime}}}{t^{\prime}}\right) t^{\prime} d \tau
$$

For this purpose let the momentum associated with the time $t$ denoted as $p_{t} \equiv p_{N+1}$ be formed as

$$
\begin{gathered}
p_{t}=\frac{\partial\left(L t^{\prime}\right)}{\partial t^{\prime}}=L-\left(\sum_{i=1}^{4} \frac{\partial L}{\partial \dot{q}_{i}} \frac{q_{i}^{\prime}}{t^{\prime 2}}\right) t^{\prime} \\
p_{t}=L-\sum_{i=1}^{4} p_{i} \dot{q}_{i} \\
p_{t}=-\left(\sum_{i=1}^{4} p_{i} \dot{q}_{i}-L\right)
\end{gathered}
$$

The last Eq.79 is exactly the total energy of the system. This leads to the conclusion that the momentum associated with the time is the negative of the system total energy [29]. That is, every system is conserved when the time is considered a mechanical variable. In other words

$$
p_{t}=-E_{\text {TOTAL }}
$$

Noting that the momentum associated with the time as a mechanical variable has the units of work, considering that the system is expanding such that it has a total net volume $\Delta V$ and by dividing both side of (15) by $V$, one obtains

$$
\frac{p_{t}}{V}=-\frac{E_{T O T A L}}{V}
$$

Recalling that the work of the pressure done on the system as its volume changes is defined as $\mathrm{P}=W / V$, then (81) can be expressed as

$$
\mathrm{P}=-\rho
$$

The last equation is equivalent to Eq.69. The importance of Eq.82 is that it has the following physical interpretation: the momentum associated with time per unit volume (the pressure associated with time) is equal to the negative of energy density that is the negative force behind the expansion of the universe.

Proof 5 (Euler-Lagrangian Equation): in addition to the previous analysis that has proved that the momentum associated with time is the dark energy, the objective now is to show that the flow of dark energy of the expanding universe is the momentum associated withe time. The Euler-Lagrangian equation takes the following form in case of a 4-D space-time world, which depends on the coordinates: $x=q_{1}, y=q_{2}, z=q_{3}, t=q_{4}$,

$$
\operatorname{div} \boldsymbol{P}_{\text {Total }}=\frac{\partial p_{1}}{\partial q_{1}}+\frac{\partial p_{2}}{\partial q_{2}}+\frac{\partial p_{3}}{\partial q_{3}}+\frac{\partial p_{4}}{\partial q_{4}}=0
$$

where $p_{i}$ is the momentum associated with coordinate $q_{i}$. When dealing with a particle, only $t=q_{4}$ is considered, and (83) is reduced to

$$
\frac{\partial p_{4}}{\partial q_{4}}=0
$$

Eq.84 implies that the time momentum is constant which by the virtue of (80) is interpreted as the system total energy, that is

$$
p_{4}=-E_{\text {Total }}=\text { const. }
$$

Eq.84 suggests that $p_{4}$ is not a function of the time coordinate $t=q_{4}$.

Now by the virtue of Neother's Invariant Theorem and Divergence Transformation Theorem of Gauss [29-31], (84) can be interpreted as follows:

$$
\iiint_{V} \operatorname{div} \boldsymbol{P} \mathrm{d} V=\iint_{S} \boldsymbol{P} \cdot \boldsymbol{n} \mathrm{d} A
$$

where $\boldsymbol{P}$ is the momentum vector of the 3 -domensional space, $\boldsymbol{n}$ is the normal vector to the surface $S$ and $d V$ is the segment of volume $V$. Now by considering that the momentum of time (as a mechanical variable) is constant and equals to the negative of the system total energy as in (85), then (86) can be modified as

$$
\iint_{S} \boldsymbol{P} \cdot \boldsymbol{n} d A+\frac{d}{d q_{4}} \iiint_{V} p_{4} d V=0
$$

or

$$
\iint_{S} \boldsymbol{P} \cdot \boldsymbol{n} \mathrm{d} A=-\frac{\mathrm{d}}{\mathrm{d} q_{4}} \iiint_{V} p_{4} \mathrm{~d} V
$$

The physical interpretation of (88) is as follows: the flux of energy (work flow) flowing through the boundary surface of the expanding space-time universe is equal to the negative of the universe total energy (the momentum associated with time): $\mathrm{P}=-\rho$. In sense of the previous analysis an equivalent equation can be drawn from (83) as:

$$
\operatorname{div} \boldsymbol{P}=-\frac{\partial p_{4}}{\partial q_{4}}
$$

Proof 6 (Einstein's Equation of General Relativity): The main goal of this proof is to show that Einstein's Cosmological constant Lambda is equivalent to the dark energy by applying Einstein's Equation of General Relativity. One of the candidates for dark energy is vacuum energy, or the Einstein cosmological constant, characterized by a pressure equal to the negative of the energy density $[9,10]$ :

$$
R_{\mu v}-\frac{1}{2} R g_{\mu v}+\Lambda g_{\mu v}=8 \pi G T_{\mu v}
$$

where the left-hand side of Einstein's equation charac- 
terizes the geometry of the space-time and the right-hand side represents the energy sources, $g_{\mu v}$ is the spacetime metric, $R_{\mu v}$ is the Recci tensor, $R$ is the spacetime curvature scalar, $\Lambda$ is a free parameter called Einstein's cosmological constant (which equivalent to the dark energy), $G$ is the Newtonian Gravitational Universal Constant and $T_{\mu \nu}$ is the energy-momentum tensor. If the vacuum (space-time) is Lorentz-invariant, then the energy momentum will take the form

$$
T_{\mu v}^{v a c}=-\rho_{v a c} g_{\mu v}
$$

where $\rho_{v a c}$ is the vacuum density (dark energy). Lorentz-invariant energy momentum is associated with an negative isotropic pressure such that

$$
\mathrm{P}_{v a c}=-\rho_{v a c}
$$

where $\rho_{v a c}$ is the vacuum pressure. It can be proved that Einstein's cosmological constant $\Lambda$ is proportional to dark energy so that

$$
\rho_{v a c}=\frac{3 c^{2}}{8 \pi G} \Lambda
$$

The physical interpretation of (93) is that the dark energy density constant Lambda is equivalent to the cosmological constant Lambda by the constants $3 c^{2} / 8 \pi G$.

Proof 7 (Einstein's Mass-Energy Equivalence Principle): consider that the universe has a total energy $E_{\text {Total }}$ with an equivalent mass $M_{E q}$, then by the virtue of Einstein's Mass-Energy Equivalence Principle, the relation is given as

$$
E_{\text {Total }}=M_{E q} c^{2}
$$

where $c$ is the speed of light. By dividing the last equation by the universe volume, one gets

$$
\frac{E_{\text {Total }}}{V}=\frac{M_{E q}}{V} c^{2}
$$

or

$$
\rho=\rho_{E q, \text { mass }} c^{2}
$$

According to Eqs.69, 71, 73, 88 and 92: $\mathrm{P}=-\rho$, then

$$
\mathrm{P}=-\rho_{E q, \text { mass }} c^{2}
$$

where $\rho_{E q, \text { mass }}$ is the equivalent dark energy mass density. Eq.96 shows that the pressure of the dark energy is equal to the negative of the equivalent dark energy mass density times the square of the light speed.

According to the previous proofs, the fourth law of thermodynamics is proposed and stated as follows: Proposed Fourth Law of Thermodynamics: "Considering time as mechanical variable, for a closed system with moving boundaries composed of homogenous isotropic cosmic fluid, the system will have a negative pressure equal to the energy density that causes the system to expand at an accelerated rate. Moreover, the momentum associated with time is equal to the negative of the system total energy" [22].

\section{COSMOLOGICAL CONSTANT AND UNIVERSE MASS-ENERGY DENSITIES}

Based on the observation that the universe is flat (WMAP), and previous proofs that the dark energy is a property of the space-time itself and it is homogenous and uniformly distributed over the universe and behaves like an ideal perfect cosmic fluid. Considering time as a mechanical variable, and based on the definition of the total kinetic energy of universe

$$
K=\frac{1}{2} M_{e q}\left(\frac{d s}{d t}\right)^{2}
$$

where $M_{e q}$ is the equivalent mass of the universe and $d s$ is the line-element of the configuration space, and $t$ stands for time. Since the kinetic energy of the universe is defined as $K=E_{\text {total }}-V$ where $V$ is the potential energy, and defining

$$
d t=\left(\frac{d t}{d \tau}\right) d \tau
$$

where $\tau$ is a cosmic space-time parameter that represents the coupling in the space-time and has the units of $m$.sec, taking into the account the Hubble expansion parameter, then the rate of change of time as a mechanical variable with respect to the coupled space-time cosmic parameter using (97) can be given as

$$
\frac{d t}{d \tau}=\frac{\sqrt{M_{e q}}}{\sqrt{2\left(E_{\text {total }}-V\right)}}\left(\frac{d s}{d \tau}\right)
$$

Now assuming for a flat universe (as in WMAP) a zero reference potential energy $V=0$, and considering the well-known equation of Einstein $E_{\text {total }}=M_{e q} c^{2}$, where $c$ is the speed of light. Then, Eq.34 can be reduced to

$$
\frac{d t}{d \tau}=\frac{1}{\sqrt{2}} \frac{1}{c}\left(\frac{d s}{d \tau}\right)=0.7071 \frac{1}{c}\left(\frac{d s}{d \tau}\right)
$$

Since the universe is expanding and Hubble parameter is a measure of this expansion, then $d s / d \tau$ is equivalent to the Hubble parameter $H$ and have the units of $(\mathrm{m} / \mathrm{s}) / \mathrm{m}$ or $1 / \mathrm{sec}$. The value of the present Hubble parameter $H_{0}$ considered is $70(\mathrm{~km} / \mathrm{s}) / \mathrm{Mpc}$ which is equivalent to $2.3 \times 10^{-18}(\mathrm{~m} / \mathrm{s}) / \mathrm{m}$ based on the WMAP 2006. Then Eq.100 yields to 


$$
\frac{d t}{d \tau}=0.7071 \times\left(0.7673 \times 10^{-26}\right) \mathrm{sec} /(\mathrm{sec} \cdot m)
$$

Since $d t / d \tau=0.54265 \times 10^{-26} \mathrm{~m}^{-1}$ has the units of $m^{-1}$ and since Einstein's Cosmological constant Lambda has the units of $m^{-2}$ in (90) and (93), then $(d t / d \tau)^{2}$ is equivalent to Lambada, that is $\Lambda=0.29447 \times 10^{-52} \mathrm{~m}^{-2}$ which complies with the astronomical observation that estimated its value $\sim 10^{-52} \mathrm{~m}^{-2}$. Now it is possible to modify Einstein's Cosmological Constant to have the form

$$
\Lambda=(d t / d \tau)^{2}=\frac{8 \pi G}{3 c^{2}} \rho_{\Lambda}
$$

then,

$$
\rho_{\Lambda}=\frac{3 c^{2}(d t / d \tau)^{2}}{8 \pi G}
$$

where $\rho_{\Lambda}$ is the density of the cosmological constant and $G$ is the Universal Gravitation Constant and has the value $6.67 \times 10^{-11} \mathrm{~N} \cdot \mathrm{m}^{2} / \mathrm{kg}^{2}$. According to (103), the universe dark energy density has the value $\rho_{\Lambda}=$ $1.2622 \times 10^{-26} \mathrm{~kg} / \mathrm{m}^{3}=6.8023 \mathrm{GeV}$. It can be concluded that rate of change of time with respect to the cosmological coupled parameter $\tau$ is behind the expansion of the universe and, accordingly, represents a physical meaning of the cosmological constant. By this, the second issue raised above has been solved. This model solves the 120-orders-of-magnitude discrepancy between the theoretical and observed values of the cosmological constant.

As it can be seen from the previous analysis, universe dark energy density is $\rho_{\Lambda}=1.2622 \times 10^{-26} \mathrm{~kg} / \mathrm{m}^{3}$ as calculated theoretically by the proposed model which totally agrees with the astronomical observations that estimate the dark energy density in the range of $\sim 10^{-26} \mathrm{~kg} / \mathrm{m}^{3}$. Furthermore, the energy density of each component $\rho_{i}$ of the universe is characterized by its density parameter $\Omega_{i}$ and the critical density $\rho_{c}$ as

$$
\Omega_{i}=\frac{\rho_{i}}{\rho_{c}}
$$

Recent observation of Supernovae by WMAP shows that $\Omega_{\Lambda} \cong 0.70, \Omega_{\text {matter }} \cong 0.30, \Omega_{\text {radiation }} \cong 5 \times 10^{-5}$. Now benefiting from (103) and (104), the average critical density of the universe is $\rho_{c}=1.8069 \times 10^{-26} \mathrm{~kg} / \mathrm{m}^{3}=$ $9.7378 \mathrm{GeV}$ which is very close to $0.74 \times 10^{-26} \mathrm{~kg} / \mathrm{m}^{3}$ estimated by cosmological observations. According to the proposed model in this paper the average density of the matter in the universe is $\rho_{m}=0.54207 \times 10^{-26} \mathrm{~kg} / \mathrm{m}^{3}=$ $2.9213 \mathrm{GeV}$. By this, the third issue raised above has been solved as well. Finally, the average density of the radiation in the universe is $\rho_{r}=2.7103 \times 10^{-31} \mathrm{~kg} / \mathrm{m}^{3}=$ $1.4558 \mathrm{MeV}$.

\section{NEW FORMULATION OF KEPLER'S LAWS: DARK ENERGY AND OUR SOLAR SYSTEM}

This section presents a new formulation of Kepler's Laws in terms of Dark Energy. Kepler's complete analysis of planetary motion is summarized in three statements known as Kepler's laws as follows: Kepler's First Law: All plants move in elliptical orbits with the Sun at one Focus. Kepler's Second Law: The radius vector drawn from the Sun to a planet sweeps out equal areas in equal time intervals. Kepler's Third Law: The square of the orbital period of any planet is proportional to the cube of the semi-major axis of elliptical orbit.

\subsection{New Interpretation of Kepler's Second Law}

The New Newtonian Kepler's Second Law: "For a planet orbiting the Sun, the rate of change of the area swept by the planet with respect to the cosmic coupled space-time parameter is proportional to the square root of the Einstein's cosmological constant'.

Proof: Kepler's Second law can be derived as a sequence of the principle of conservation of angular momentum. Consider a planet of mass $M_{P}$ moving about the Sun in an elliptical orbit. Let the planet to be considered as a system and the Sun is so heavy so that it does not move relatively to the planet. The gravitational force acting on the planet is a central force, always along the radius vector and directed toward the Sun. Taking the torque on the planet due to this central force which is always zero because the central force $F$ is parallel to $R$. That is

$$
\mathrm{T}=R \times F=0
$$

Recalling that the time rate of change of the angular momentum of the system equals the external net torque on the system; that is, $\mathrm{T}=d L / d t$, where $L$ is the angular momentum. Therefore, and because torque $\mathrm{T}=0$, the angular momentum $L$ of the system (the planet) is a constant

$$
L=R \times P=M_{P} R \times v=\text { const } .
$$

By the geometric of the motion, (106) can be modified as follows. In a time interval $d t$, the radius $R$ sweeps out the area $d A$, which equals half the area $|R \times d R|$ of the parallelogram formed by the vectors $R$ and $d R$. Because the displacement of the planet is the time interval $d t$ is given by $d R=v d t$, one gets

$$
d A=\frac{1}{2}|R \times d R|=\frac{1}{2}|R \times v d t|=\frac{L}{2 M_{P}} d t=K_{A} d t
$$

By dividing both sides of Eq.107 by the coupling pa- 
rameter variation $d \tau$ yields

$$
\frac{d A}{d \tau}=\left(\frac{L}{2 M_{P}}\right) \frac{d t}{d \tau}=K_{A} \frac{d t}{d \tau}
$$

By the virtue of (101) the value of $d A / d \tau$ is proportional to square root of Einstein's Constant, i.e., $0.54265 \times 10^{-26}$. Note that $d A / d \tau$ is a vector and has the units of velocity and directed upward, perpendicular to the Sun-planet plane and along the angular momentum vector. From vectors view point and as a result of the rate change of time with respect to the coupled space-time parameter $\tau$ and the angular motion of the plant around the Sun, the planet tends to move in a vertical direction perpendicular to the elliptic plane. Consequently, the planet tends to leave the plenary motion, but the existence of the dark energy with a very small value ( $\rho_{\Lambda}=$ $1.2622 \times 10^{-26} \mathrm{~kg} / \mathrm{m}^{3}$ ) damps such an upward motion and keeps it so small comparing to the planetary rotational velocity. That is why a stable circular path is always obtained and planets always move in a stable co-plenary motion.

Calculations results of such a model shows that in the case of the Erath-Sun system, the vertical motion of the Erath is approximately $1.5 \times 10^{-11} \mathrm{~m} / \mathrm{sec}$ which is very negligible comparing to its rotational velocity. Moreover, in case of the Mercury-Sun system, the Mercury vertical velocity is in the range of $2.5 \times 10^{-12} \mathrm{~m} / \mathrm{sec}$. Meanwhile, such a vertical motion of the Sun orbiting our Milky Way Galaxy is approximately close to $2.5 \times 10^{-31} \mathrm{~m} / \mathrm{sec}$. According to the previous analysis, then, it can be seen that Einstein's Constant (and dark energy) is behind the stability of elliptic orbits. For given planet orbiting the sun, the momentum is constant, its mass is constant, then the swept area is maintained fixed due to Einstein's Constant Lambda and constant dark energy density at given orbital location.

\subsection{New Interpretation of Kepler's Third Law}

The New Newtonian Kelper's Third Law: "The square of the orbital period of any planet orbiting the Sun is inversely proportional to the dark energy density at that orbit'.

Proof: Consider a planet of mass $M_{P}$ that is assumed to be moving about the Sun with mass $M_{S}$ in a circular orbit. Because of the gravitational force provides the centripetal acceleration of planets as it moves in a circle. Using Newton's second law for an object in uniform circular motion then,

$$
\frac{G M_{S} M_{P}}{R^{2}}=\frac{M_{P} v^{2}}{R}
$$

The orbital speed of the planet is $2 \pi R / T$, where $T$ is the period; then (109) becomes

$$
\begin{gathered}
\frac{G M_{S}}{R^{2}}=\frac{(2 \pi R / T)^{2}}{R} \\
T^{2}=\left(\frac{4 \pi^{2}}{G M_{S}}\right) R^{3}=K_{S} R^{3}
\end{gathered}
$$

where $K_{S}=\left(\frac{4 \pi^{2}}{G M_{S}}\right)=2.97 \times 10^{-19} \mathrm{sec}^{2} / \mathrm{m}^{3}$ for our solar system. Eq. 111 is still valid if we replace the radius $R$ with the length of the semi-major axis for elliptical orbits. Note that the constant is inversely proportional to the mass of the central body (the Sun in our case) and independent of the mass of the planet.

By the virtue of Eq.111, the square of the orbital period of any planet is proportional to the cube of the semi-major axis of elliptical orbit; that is $T^{2} \alpha R^{3}$. In other words, it is a volumetric measure. Now since Dark Energy is very homogeneous and uniformly distributed all over the universe, then it is very reasonable to calculate the dark energy density consisted in sphere centred at the Sun and have radius $R$. To do so, multiply both sides of Eq.111 by $(4 / 3) \pi$ to obtain

$$
\frac{4}{3} \pi T^{2}=\frac{4}{3} \pi K_{S} R^{3}
$$

Now assume that the equivalent mass of the dark energy contained in that sphere is $M_{e q}$, then by dividing both sides of (112) by this equivalent mass $M_{e q}$ yields

$$
\frac{1}{M_{e q}}(4 / 3) \pi T^{2}=K_{S} \frac{1}{M_{e q}}(4 / 3) \pi R^{3}
$$

The expression $\frac{1}{M_{e q}}(4 / 3) \pi R^{3}$ represents the reciprocal of the dark energy density $\rho_{E}$ in $\mathrm{kg} / \mathrm{m}^{3}$. According to this analysis (113) can be rewritten as

$$
T^{2}=\frac{K_{S} M_{e q}}{(4 / 3) \pi} \frac{1}{\rho_{E}}=K_{E} \frac{1}{\rho_{E}}
$$

where

$$
\begin{aligned}
K_{E} & =\frac{K_{S} M_{e q}}{(4 / 3) \pi}=\left(\frac{3 \pi}{G M_{S}}\right) M_{e q} \\
& =0.709 \times 10^{-19} M_{e q} \mathrm{~kg} \cdot \sec ^{2} / \mathrm{m}^{3}
\end{aligned}
$$

for our solar system.

The heavier the central body is, the more dark energy is dragged to it. It is then very possible to estimate the value of dark energy density by knowing the orbiting period. Calculation results show that dark energy density at locations close to the earth is $0.25 \times 10^{-25} \mathrm{~kg} / \mathrm{m}^{3}$. 
Meanwhile, it is in the range of $18 \times 10^{-25} \mathrm{~kg} / \mathrm{m}^{3}$ for Mercury which agrees with the observations. The more the planets are close to Sun, the faster the orbiting speed will be. This is due to the dark energy density which behaves as a perfect fluid. Hence, more of dark energy fluid exits near to the sun.

According to the previous that shows a fluidic nature of the dark energy, then new physical interpretation of Kepler's First Law is as follows: The New Newtonian Kepler's First Law: "Revolving of the Sun around itself causes elliptic streamlines in the fabric of the cosmic space-time fluid on which planets orbit".

\section{THE ULTIMATE FATE OF THE UNIVERSE: THE BIG IMPULSE}

This part presents a parametric modeling of the ultimate fate of the universe. Such a model is based on: the Big Bang theory, Einstein's General Relativity, Friedmann-Robertson-Walker model, Lambda-Cold Dark Matter model (CDM), Hubble expansion parameter, Cosmic Microwave Background radiation (CMB), Hubble Ultra Deep Field (HUDF) observation of the deep space, Wilkinson Microwave Anisotropy Probe (WMAP) [22,23], the exponential inflation model, cosmic horizon problem, breaking symmetry model [27], the estimation of the age of the universe by 13.7 billion years, the NASA's observation of dark energy that caused the expansion of the universe 9 billion years ago and before that time the expansion was decelerating.

Based on all of that and on the fact that what we are observing is just the history of our universe, a secondorder (parabolic) parametric model is obtained in this proposed paper. Such a parabolic model describes the rate of change of time with the space-time cosmic coupled parameter, that is, the expansion of the universe. This model shows that the universe is approaching the universe cosmic horizon line and will pass through a critical point that will influence significantly its fate. Considering the breaking symmetry model and considering a very infinitesimally period of time similar to that of the inflation $10^{-33} \mathrm{sec}$ happened early in the universe, then the universe will witness an infinitesimally stationary state by the virtue the variational principle of mechanics and as result of that the a very massive impulse will occur and correspondingly the universe will collapse. The physical meaning of such a model is that as the universe time (clock) stops, the universe will collapse.

Proof: Considering time as a mechanical variable, and based on the definition of the total kinetic energy of universe as

$$
\frac{d t}{d \tau}=\frac{1}{\sqrt{2}} \frac{1}{c}\left(\frac{d s}{d \tau}\right)=0.7071\left(\frac{1}{c} \frac{d s}{d \tau}\right)=f(\tau)
$$

Based on the fact that our observation of the universe is a record of its history, based on the cosmic exponential inflation model that universe brought back to our observable horizon within $10^{-33} \mathrm{sec}$ after the Big Bang, based on the Hubble observation that after the cosmic inflation the universe expansion decelerated for 4.7 billion years then it has started to accelerate up until these days for 9 billion years, taking into account of the age of the universe is 13.7 billion years by CDM model, based on Hubble Ultra Deep Field (HUDF) observation of the deep space, based on the astronomical observations using Cosmic Microwave Background radiation (CMB) of the photons signals had sent to us 300,000 years after the Big Bang that shows that the universe is observable within the cosmic horizon, it can be concluded that the universe is still within the observable horizon line, then, a second order parabolic parametric model is proposed to describe the expansion behavior of the universe.

This curve is open up and bounded by the cosmic horizon line, and the left portion of the curve accounts for the deceleration of the expansion of the universe for the first 4.7 billion years, meanwhile, the right portion of this parabolic curve accounts for the acceleration of the universe expansion for 9 billion years. Moreover, this model intersects with the cosmic horizon line at the first critical breaking symmetry point within the cosmic inflation, that is, the Big Bang. The minimum point (turning point between deceleration and acceleration) is characterized in terms of Hubble parameter when the universe starts accelerating at $\sim 10^{-18} \mathrm{sec}^{-1}$ rate. Furthermore, the second intersection point of this parametric parabolic curve with the universe cosmic horizon line represents the second breaking symmetry critical point at which our proposed Big Impulse occurs and would be estimated as follows.

$$
\frac{d t}{d \tau}=\left(a \tau^{2}-b \tau+c\right)
$$

The constant $a, b$, and $c$ are constants to be determined according to the initial and given condition. It is clear that the constant $c$ is zero since the $d t / d \tau=0$ at $\tau=0$. Now the model (116) is reduced to

$$
\frac{d t}{d \tau}=\left(a \tau^{2}-b \tau\right)=a\left(\tau^{2}-\frac{b}{a} \tau\right)
$$

It is very useful now to write (117) in completing-thesquare form because it can represent the minimum point and if the curve is open-up in a simple way

$$
\frac{d t}{d \tau}=a\left(\tau-\frac{1}{2} \frac{b}{a}\right)^{2}-\frac{1}{4} \frac{b^{2}}{a}
$$

Note that the vertex point is $\left(\tau_{c}, H_{\min }\right)$, where $H_{\min }$ represents the Hubble parameter when the universe starts to accelerate 9 billion years go $\sim 10^{-18} \mathrm{sec}^{-1}$, and $\tau_{c}$ is 
its corresponding space-time parameter value. Note also that $t \sim \tau^{3}$, then at 4.7 billion years, $\tau \sim 1.6751$. Since $\tau_{c}=\frac{1}{2} \frac{b}{a}=1.6751$, then

$$
\frac{b}{a}=3.3502
$$

From the vertex-form (completing the square),

$$
H_{\min }=\frac{1}{4} \frac{b^{2}}{a}=\frac{1}{4}\left(\frac{b}{a}\right) b
$$

or

$$
b=\frac{4}{(b / a)} H_{\min }
$$

It can be seen that the ratio $b / a=3.3502$ is not a function of $H_{\min }$ contrary to the constant $b$ which a function of $H_{\min }$ as given in (121). Solving for $a$ and $b$ in (119), (120) and (121), one obtains $b=1.194 H_{\text {min }}$ and $\mathrm{a}=0.3564 H_{\min }$. Since the constant $a$ is positive then the second order parabolic curve is open up.

Substituting for $a$ and $b$ in the expansion parabolic parametric Eq.117 yields

$$
\frac{d t}{d \tau}=H_{\min }\left(0.3564 \tau^{2}-1.194 \tau\right)
$$

Simulation of the parametric expansion (122) with respect to $\tau$ in Figure 6 shows the Big Bang singularity, the left portion of the decelerated expansion, then the universe started to accelerate at $\tau \sim 1.6751$ and heading up towards the cosmic horizon and the breaking symmetry critical point.

Since the Hubble parameter is a slowing down parameter and since it is characterized by $\sim 10^{-18} \mathrm{sec}^{-1}$ when the universe starts to accelerate, it should be mentioned that Hubble parameter is not a constant but it is time changing and slowly decreasing with respect the universe age. Eq.120 shows that the constant $b$ is Hubble Parameter dependent and because Hubble parameter recently is slower than of that one 9 billion years ago, then it should be modified as

$$
b^{*}=1.194 H_{\min } k
$$

where $k$ is s correction factor to account for the decrease in the value of Hubble parameter over ages. The most interesting value of it is when $k=0.7071=1 / \sqrt{2}$ as in (120) which leads to the correspondent parabolic parametric expansion equation

$$
\frac{d t}{d \tau}=H_{\min }\left(0.3564 \tau^{2}-0.8443 \tau\right)
$$

Analyzing Eq.124 shows that it has one root at zero singularity (Big Bang) and another root at $\tau=2.369$ which is corresponding to $t=13.3295$ billion years since $t \sim \tau^{3}$ which is very close to the estimations of the age of the universe. Remember that the LCDM model estimates the age of the universe by $13.7+/-0.2$ billion years. Other astronomical calculations estimate the age of the universe between 13-14 billion years. Simulation of the parametric expansion (124) with respect to $\tau$ in Figure 7 shows the Big Bang singularity, the left portion of the decelerated expansion, then the universe started accelerating at $\tau \sim 1.6751$ heading up towards the cosmic horizon and will cross it at the second critical point $\tau=2.369$ as a breaking symmetry critical point.

Assuming now that the universe started its acceleration 8.85 billion years ago, that is at the age 4.85 billion years, then the constants in the parametric equation are as follows. At 4.85 billion years $\tau \sim 1.6927$, and

$$
\begin{aligned}
& \tau_{\min }=\frac{1}{2} \frac{b}{a}=1.6927, \frac{b}{a}=3.3854, \\
& b=\frac{4}{(b / a)} H_{\min }=1.1815 H_{\min }, a=\frac{b}{3.3854}=0.349,
\end{aligned}
$$

and considering $k=0.7071$, then the parametric equation will take the following form

$$
\frac{d t}{d \tau}=H_{\min }\left(0.349 \tau^{2}-0.8354 \tau\right)
$$

Eq.125 will have two roots at the zero singularity (the Big Bang) and another root at $\tau=2.3937$ which is corresponding to $t=13.7154$ billion years since $t \sim \tau^{3}$. Such value is very close to the current estimated value of the age of the universe using Hubble parameter and LCDM model of $13.7+/-0.2$ billion years. Simulation of the parametric expansion (125) with respect to $\tau$ in Figure 8 shows the Big Bang singularity, the left portion of the decelerated era, then the universe started to accelerate at $\tau_{c}=1.6927$ and heading up towards the universe cosmic horizon line where the universe is very close to the cosmic horizon line and will have a breaking symmetry critical point at $\tau=2.3937$. By zooming out the upper right portion of the parametric expansion curve it can be seen how close the universe is to the Big Impulse as shown in Figure 9 and correspondingly it will dramatically influence its fate and cause it collapse.

It should be emphasized that this parametric model cannot predict the exact time of the second critical point, that is, the Big Impulse. That is because the parametric model is based on several assumptions: 1) This model is a parametric model and is not explicitly a function of the time, 2) It is assumed that the order of the expansion parametric model is two, 3) The parametric transformation from the time scale to the parametric scale is of third order, 4) This parametric model is Hubble parameter dependent and a correction factor is assumed to account for the slowly decreasing Hubble parameter, 5) The accuracy in the astronomical observations of the age of the universe is in the range of $+/-0.2$ billion years, 6) The 
astronomical observation that the expansion of the universe as long as 9 billion years ago has also some measurement error. But in general, the model is significantly correct in terms of describing the behavior and the trend of the expansion of the universe and its closeness to the cosmic horizon and the symmetry breaking point.

Moreover, taking into account the very massive equivalent mass-energy of the universe, the very infinitesimal stationary time and principle of impulse forcemomentum principle, then the universe will experience a very massive impulse given the name the Big Impulse just to distinguish it from the Big Bang. This can be verified by the virtue of the variational principle of mechanics as follows. For the 4-diemnsional space-time, the stationary action integral (the universe expansion) would be (considering that $t^{\prime}=d t / d \tau=0$ )

$$
\delta d \bar{s}=\delta \int_{\tau_{1}}^{\tau_{2}} L t^{\prime} d \tau=0
$$

According the principle of impulse-momentum, a very massive impulse would occur as follows

$$
\begin{gathered}
F_{\text {impulse }} \Delta t=M_{\text {uinverse }, E q}\left(v_{2}-v_{1}\right)=-M_{\text {uinverse, } E q} v_{1} \\
F_{\text {impulse }}=-M_{\text {uinverse, } E q}\left(v_{1} / \Delta t\right)
\end{gathered}
$$

The negative sign shows that the line of action of the Impulse force is in the opposite of the outward expansions, that is, it would act towards the universe system. Such a Big Impulse will determine the fate of the universe and cause to break the symmetry of the universe and push it beyond the cosmic horizon. It is estimated that the mass of the universe is $3 \times 10^{50} \mathrm{~kg}$ as by Jeanne Hopkins in his article "Universe" Glossary of Astronomy and Astrophysics, Chicago: the University of Chicago, 183, 1980. A very infinitesimally period of time similar to that of the inflation $10^{-33}$ sec happened early in the universe. Calculations of (128) show that, our universe will experience an impulse force in the order $10^{83} \mathrm{~N}$. Because the impulse force will act in a direction opposite to gravitational force line of action, then, the gravitational bounding force will collapse, and so the universe will.

\section{SIMULATION RESULTS}

This section is dedicated to simulate some of the proposed models presented earlier in this paper as follows.

\subsection{Can Dark Energy Be Generated?}

Macro Scale: astronomical observations have brought the evidence that dark energy is homogenous, isotropic and uniformly fills the space with a density approximated $10^{-26} \mathrm{~kg} / \mathrm{m}^{3}$. It is known that it does not interact with forces other than gravity. For example, for a space-time sphere with $1 \mathrm{~m}$ radius, the total amount of dark energy contained in it is $4.188 \times 10^{-26} \mathrm{~kg}$ which is equivalent to $3.7699 \times 10^{-9}$ Joules $(=23.562 \mathrm{GeV})$. One Joule of dark energy is equivalent to a space-time sphere of $642.5258 \mathrm{~m}$ radius. One kilo Joules of dark energy is equivalent to a space-time sphere of $6425.3 \mathrm{~m}$ radius. Meanwhile, One Mega Joules of dark energy is equivalent to a space-time sphere of $64252 \mathrm{~m}$ radius. Furthermore, One Giga Joules of dark energy is equivalent to a space-time sphere of $642.520 \mathrm{~km}$ radius. According to this analysis it seems that massive objects are needed to drag reasonable amounts of dark energy as galaxies, stars or planets as shown in Figure 2.

Micro-Nano Scale: previous analysis is based on the macro scale level. To test now the existence of dark energy at the micro-nano level, the following experiment is proposed. This system is composed of a closed cubic box of edge length equal to $1 \mathrm{~m}$ and a micro piston-cylinder is then to be placed inside that box. Such a micro piston-cylinder might be fabricated using Micro-ElectroMechanical Systems technology (MEMS). The total amount of dark energy contained inside the cubic box is in the range of $10^{-26} \mathrm{~kg}$ which is equivalent to $0.90 \times 10^{-9}$ Joules (or $5.625 \mathrm{GeV}$ ). If the volume of the micro piston-cylinder is chosen such that it has a $1 \mathrm{~cm}^{3}$, it contains an amount of energy equivalent $10^{-32} \mathrm{~kg}$ or $9 \times 10^{-16}$ Joules or $5625 \mathrm{eV}$. Now if the micro piston-cylinder is placed inside the cubic box, then the piston will move to the left as shown in Figure 5. That is because the dark energy inside the cubic box is much higher that that inside

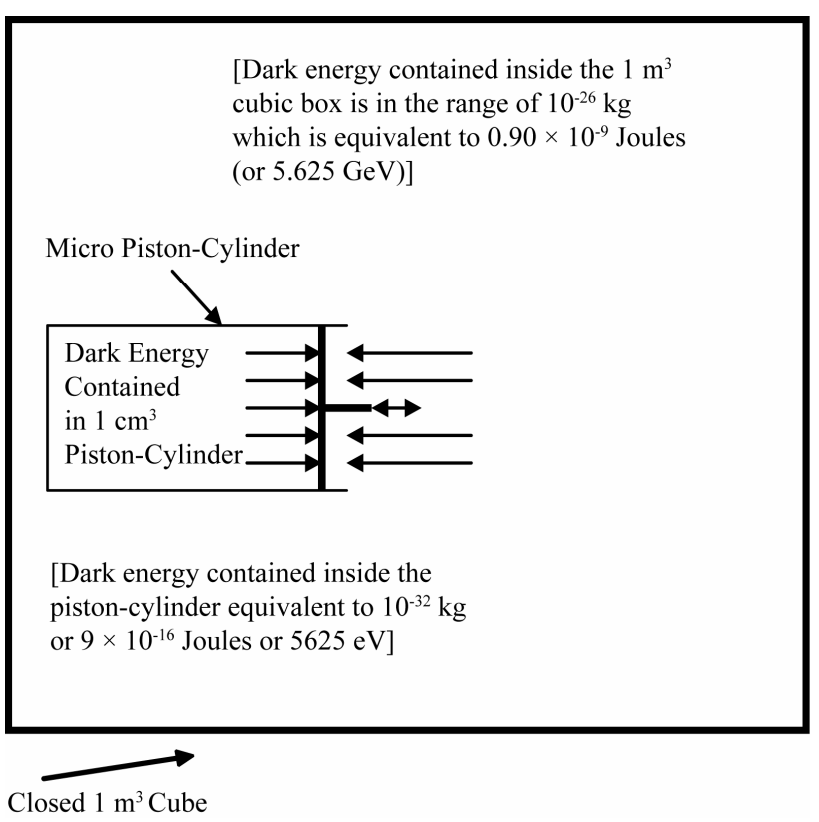

Figure 5. Micro piston-cylinder system to test the pressure work of dark energy: the piston will move by 0.18 nanometer. 


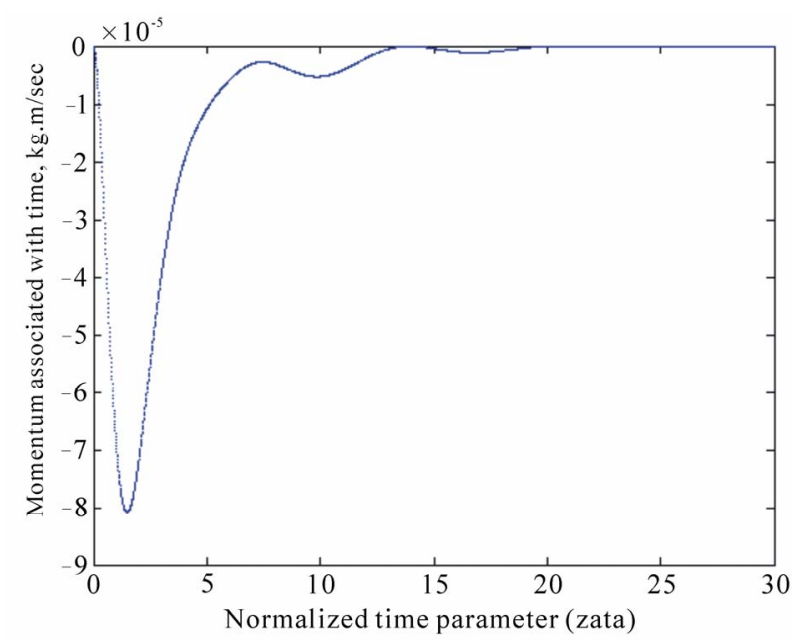

Figure 6. Dark energy associated with time as a mechanical variable of a 6 dof micro space robot $\left(10^{-5} \mathrm{j}\right)$.

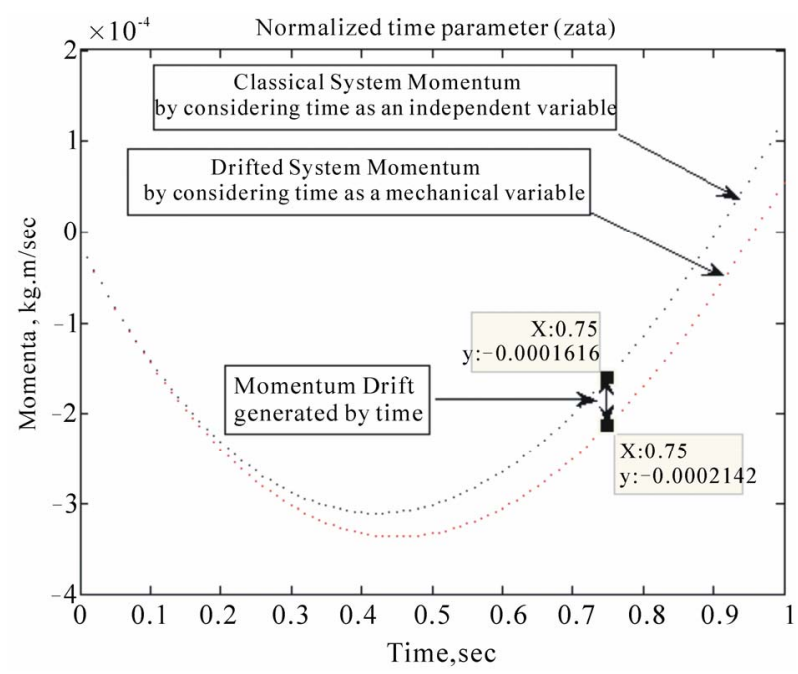

Figure 7. Drifted momentum (dark energy effect) by considering time as a mechanical variable of a 6 dof micro space robot $\left(10^{-4} \mathrm{j}\right)$.

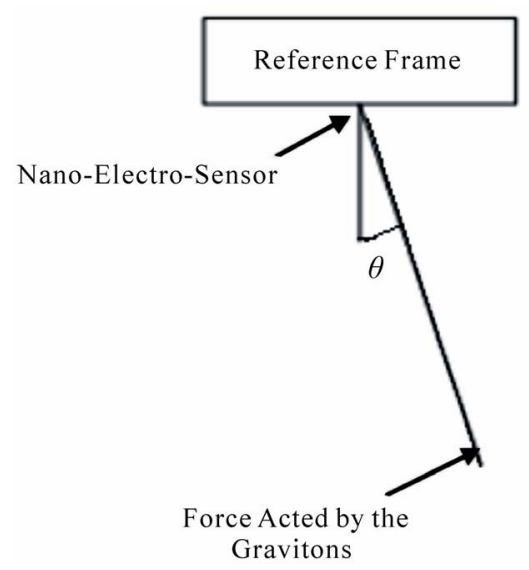

Figure 8. Micro-space antenna detector.

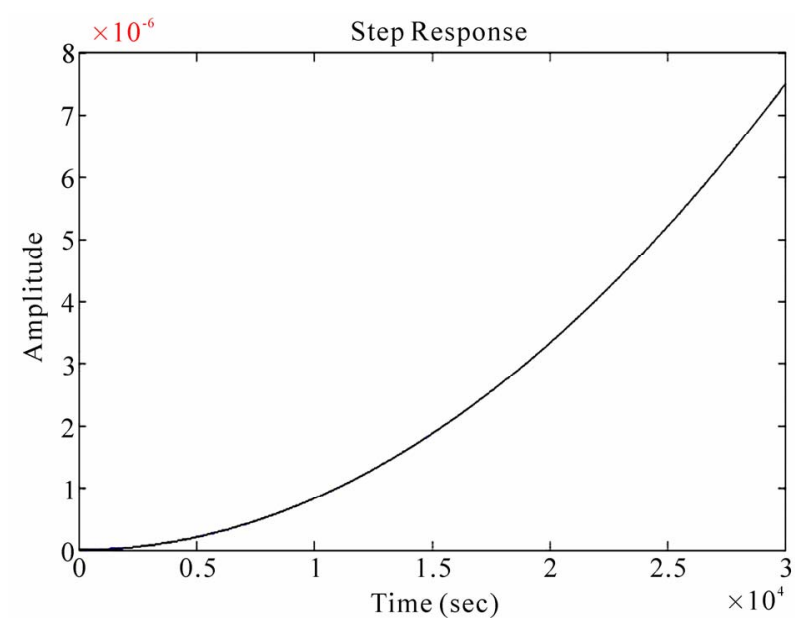

Figure 9. The micro space antenna (top), The angular position of the micro space antenna $\left(10^{-33}\right) \mathrm{rad}($ down).

the micro piston-cylinder. Such a displacement is proportional to the dark energy density. The work of the dark energy is $\delta W=-F d s=-\mathrm{P} A d s$ as in (1). According to the principle of conservation of energy, this work should be equal to the kinetic energy of the piston $K E=$ $0.5 m_{p}(d s)^{2}$. Hence, $K E=-\mathrm{P} A d s=0.5 m_{p}(d s)^{2}$, then, $d s=-2 \mathrm{P} A / m_{p}$, where $m_{p}$ is the piston mass, if the piston area is chosen as $A=10^{-4} \mathrm{~m}^{2}$, its mass is $10^{-3} \mathrm{~kg}$, and estimating the dark energy pressure as $0.90 \times 10^{-9}$ $\mathrm{J} / \mathrm{m}^{3}(5.625 \mathrm{GeV})$, then the piston will move 0.18 nanometer. By this, it is possible that dark energy can be utilized to operate mico or nano systems. Such systems can have several applications in space, biomedical or electronics applications.

\subsection{A Micro Free Flying Space Robot}

The purpose of simulation is to show the dark energy associated with a mechanical system by considering time as a mechanical variable. The dark energy is equal to the-momentum associated with time. Such energy (negative pressure) drifts the system a way. The system is composed of 6-DOF micro space robot arm and mounted on a micro base satellite and used to demonstrate the analytical results. The mass of the base servicing satellite is chosen as $10 \mathrm{~g}$, the masses of the 6-robot arm as $\left[\begin{array}{llll}1 & 1 & 1 & 1\end{array}\right.$ $11]$ g. All initial conditions are assumed to be zero. The desired values for the robot angular position are chosen as $q_{\text {des }}=\left[\begin{array}{llllll}0.3 & 0.2 & 0.1 & 0.6 & 0.5 & 0.4\end{array}\right]^{\prime}$. A PD controller is used to control the micro space-satellite robot with 0.01 value selected for both the proportional and derivative parameters. The simulation in Figure 6 shows the momentum associated with the time by considering it as a mechanical variable (dark energy), meanwhile, Figure 7 shows a comparison between the classical momentum and the drift in momentum by considering time as a me- 
chanical variable. This comparison indicates that there is indeed a drift (expansion) equal to the negative of the system total energy (dark energy). This drift is a common phenomena for free-flying space robot.

\subsection{Micro-Space Antenna Detector}

A space robotic mission can be proposed to probe the gravity waves and gravitons. The micro space antenna is located at a proper distance from the mother spacecraft. The mass of the mother spacecraft is relatively very huge comparing to the mass of the antenna. According to the previous analysis and the General Relativity, the mother spacecraft will generate a gravity field and carried out by the gravitons. The micro antenna will be deflected as a result of the graviton torque acting on it. Now, consider a space antenna shown in Figure 8, where $l$ denotes the length of the antenna and $m$ denotes to its mass. Let $\theta$ denote the angle subtended by the antenna and the vertical axis through the pivot point. Using Newton's second law of motion, we can write the equation of motion in the tangentional direction as $m l \ddot{\theta}=-b l \dot{\theta}+\frac{T}{l}$ where $T$ is the torque acted by the gravitons on the antenna. This equation will serve the simulation later. A micro space antenna model is used to verify the analysis results of gravity waves and gravitons. The mass of the antenna is chosen as $0.000150 \mathrm{~kg}$, its length chosen as $0.0004 \mathrm{~m}$. All initial conditions are assumed to be zero. It is assumed that the mother spacecraft generated a gravity field due to its huge mass and velocity comparing to the antenna. The model (29) is used in the simulation based on the assumption that the graviton is 0.707 of the mass of the photon (that $2.5119 \times 10^{-52} \mathrm{~kg}$ ). The position response of the micro space inverted antenna is shown in Figure 9. It shows that the angular position is increasing in the scale of $10^{-35} \mathrm{rad}$. Such value cannot be measured using nowadays technology. Future developments might make this measurement possible.

\subsection{Space-Time parboliod}

Simulation results in Figures 10-14 show four cases when $E_{\text {TOTAL }}=1, E_{\text {TOTAL }}>1, E_{\text {TOTAL }}<1, E_{\text {TOTAL }}=c^{2}$ for the proposed Null Paraboliod (46). Finally in Figure 15 the Minkovisky Null cone (43), respectively.

These figures compare paraboloid manifold with Robertson-Walker and Minkowski's four-dimensional world are defined as follows for a flat universe. The reduced line-element (46) represent a null paraboloid which depends on the system total energy $E_{\text {TOTAL }}$ and the maxi-mum increase. In this case if a particle is moving with the speed of light then the null rays will lie on the surface of the paraboloid.

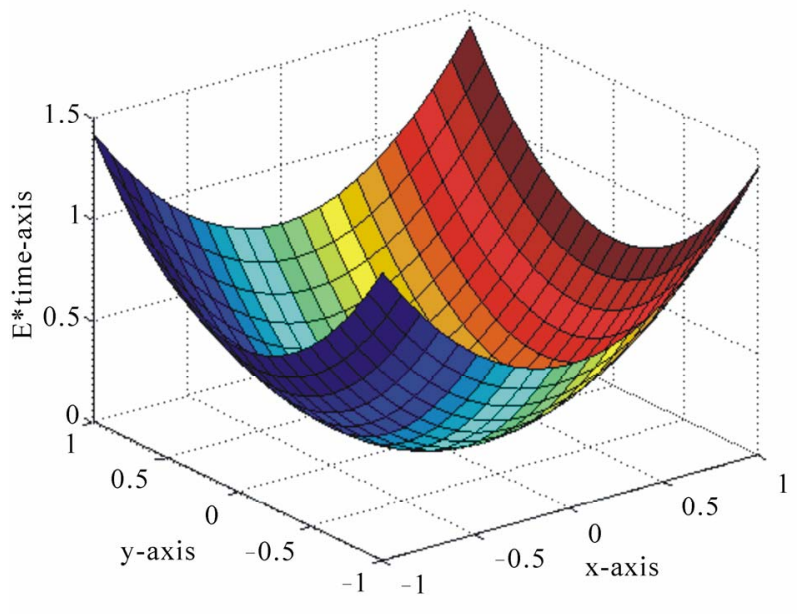

Figure 10. Null Paraboloid for $E=1$.

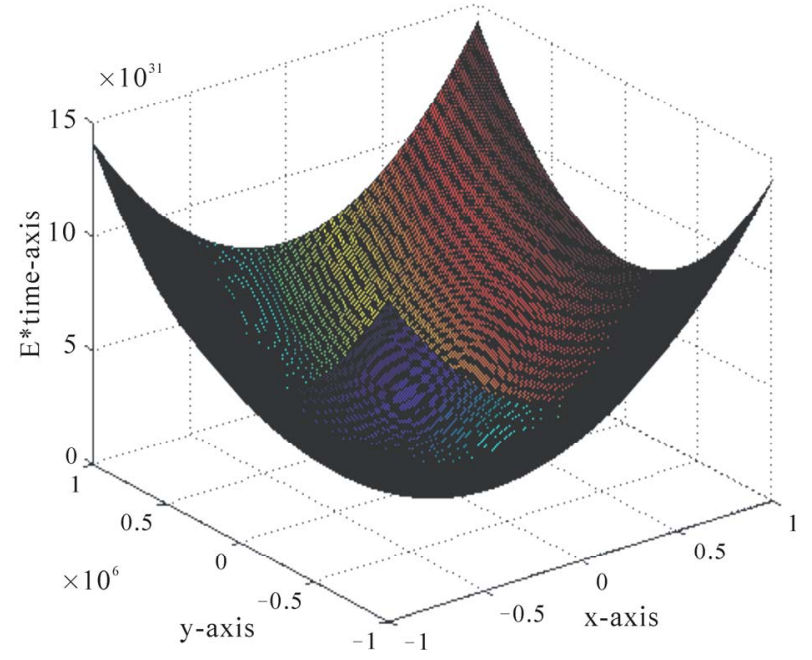

Figure 11. Null paraboloid for $E=1 \mathrm{e} 20$.

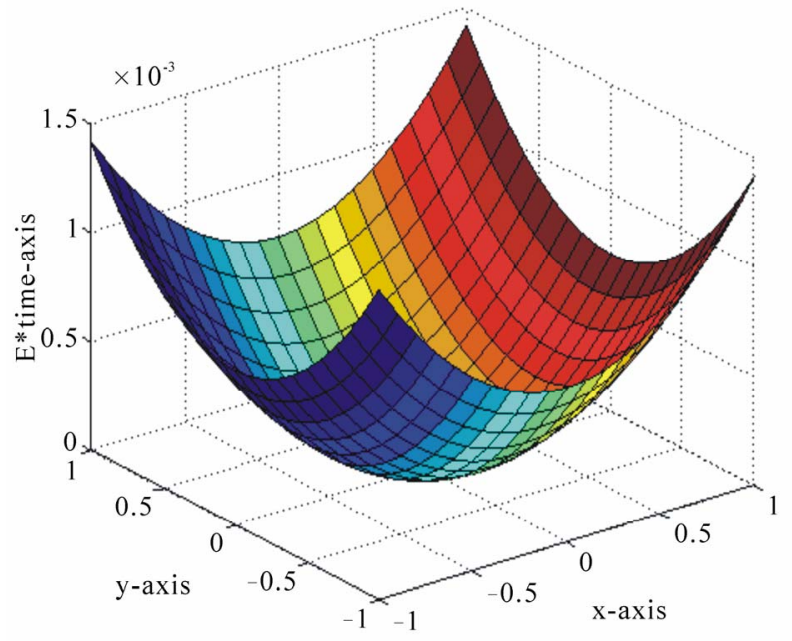

Figure 12. Null paraboloid for $\mathrm{E}=0.001$. 


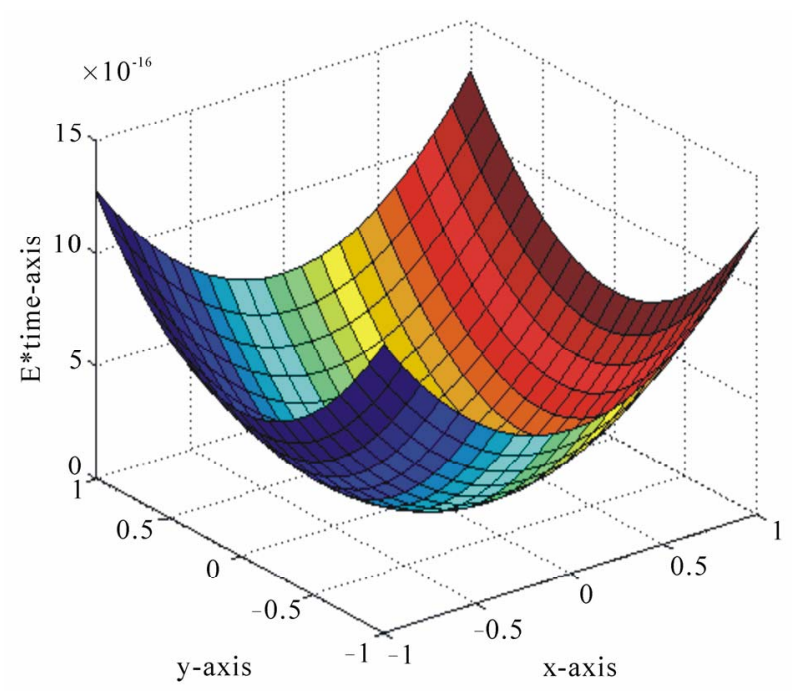

Figure 13. Null light paraboloid $E=c^{2}$.

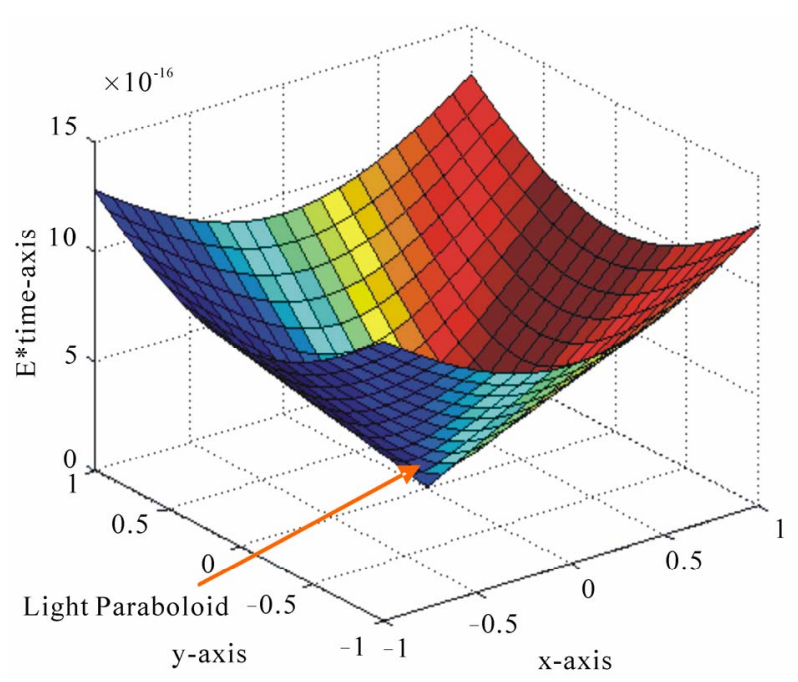

Figure 14. Minkovisky null cone $E=c^{2}$.

\subsection{The Ultimate Fate of the Universe}

Simulation of the ultimate fate of the universe in terms of the parametric expansion model is demonstrated in Figures 15-17 for Eqs.124 and 125, respectively. It can be seen that the universe is approaching to a breaking symmetry state and a Big Impulse that will cause its collapse. Such a Big Impulse will determine the fate of the universe and cause to break the symmetry of the universe and push it beyond the cosmic horizon. It is estimated that the mass of the universe is $3 \times 10^{50} \mathrm{~kg}$ as by Jeanne Hopkins in his article "Universe" Glossary of Astronomy and Astrophysics, Chicago: the University of Chicago, 183, 1980. Considering a very infinitesimally period of time similar to that of the inflation $10^{-33} \mathrm{sec}$ happened early in the universe. Calculations of (128)

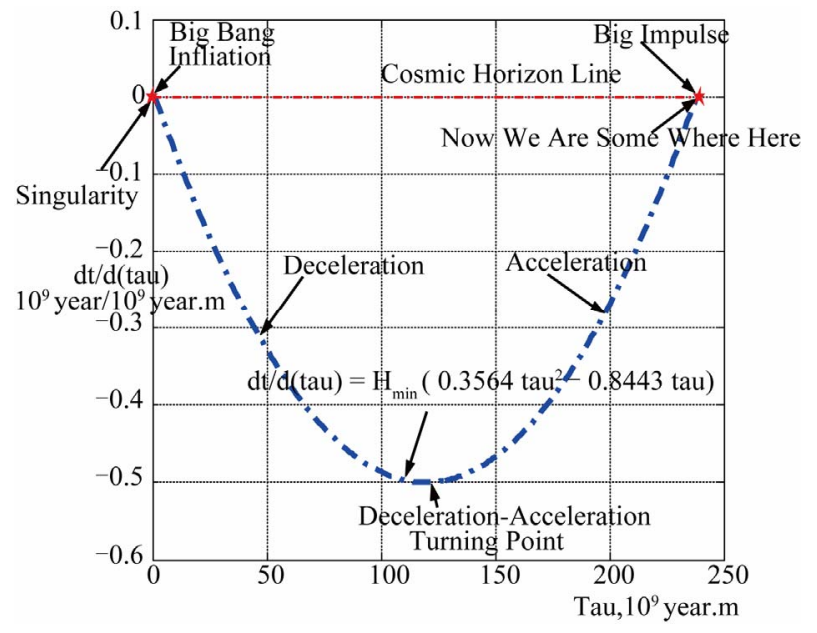

Figure 15. The expansion parametric model of the Eq.124.

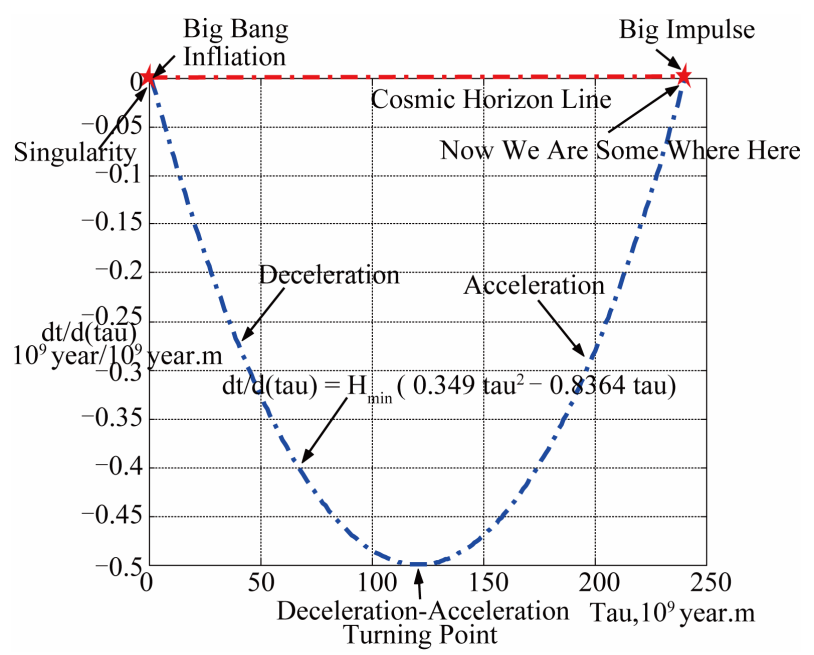

Figure 16. The expansion parametric model of Eq.125.

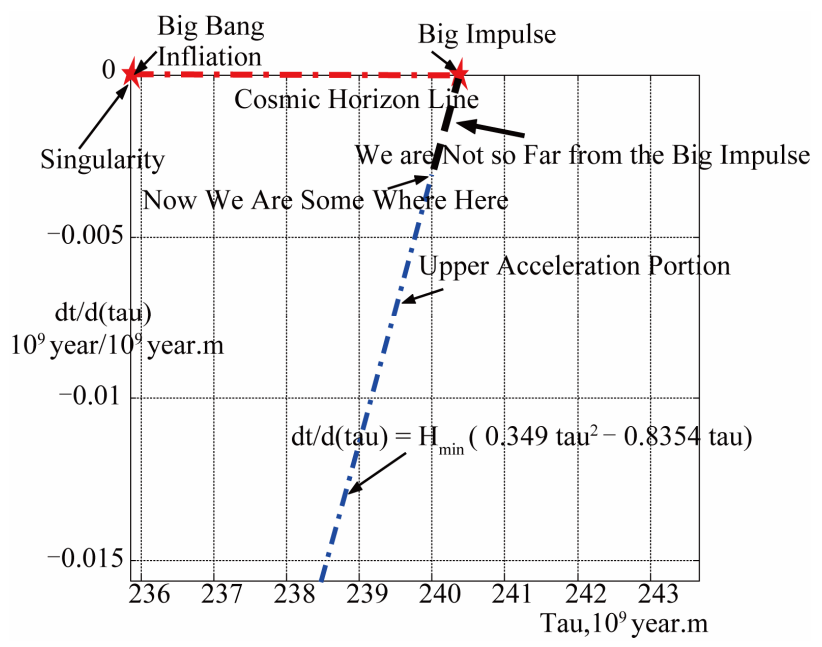

Figure 17. Zooming the upper right portion of Eq.125. 
show that, our universe will experience an impulse force in the order $10^{83} \mathrm{~N}$. Because the impulse force will act in a direction opposite to gravitational force line of action, then, the gravitational bounding force will collapse, and so the universe will.

\section{CONCLUSIONS}

This paper has presented a foundation of the theory of universe dark energy, a solution of Einstein's cosmological constant problem, physical interpretation of universe dark energy and Einstein's Cosmological Constant and its value, values of universe dark energy density, universe matter density, and universe radiation density. Einstein's Cosmological constant Lambda Lambada, is calculated as $0.74 \times 10^{-26} \mathrm{~kg} / \mathrm{m}^{3}$ which complies with the astronomical observation that estimated its value $\sim 10^{-52} \mathrm{~m}^{-2}$. The universe dark energy density has been calculated to have the value $\rho_{\Lambda}=1.2622 \times 10^{-26} \mathrm{~kg} / \mathrm{m}^{3}=$ $6.8023 \mathrm{GeV}$.The average critical density of the universe is $\rho_{c}=1.8069 \times 10^{-26} \mathrm{~kg} / \mathrm{m}^{3}=9.7378 \mathrm{GeV}$ which is very close to $0.74 \times 10^{-26} \mathrm{~kg} / \mathrm{m}^{3}$ estimated by cosmological observations. According to the proposed model in this paper the average density of the matter in the universe is $\rho_{m}=0.54207 \times 10^{-26} \mathrm{~kg} / \mathrm{m}^{3}=2.9213 \mathrm{GeV}$. By this, the third issue raised above has been solved as well. Finally, the average density of the radiation in the universe is $\rho_{r}=2.7103 \times 10^{-31} \mathrm{~kg} / \mathrm{m}^{3}=1.4558 \mathrm{MeV}$.

It is found that the momentum associated with time is behind the expansion of the universe since it is equal to the negative of the universe total energy. The negative pressure of the dark energy is equal to the energy density. It has been proved also that dark energy is a property of the space-time itself. Based on the fluidic nature of dark energy, the fourth law of thermodynamics is presented; a new formulation of the three laws of Kepler is proposed as well. A second order parametric model of the expansion of the universe is presented and estimated that the universe is acceleration to its fate and will soon experience a Big Impulse that will cause its collapse.

Moreover, in this paper a modeling approach of a symmetrical gravitational field is proposed. In the propsoed model time is treated as a mechanical dependent variable. In the proposed field tensor, the coupling in time and its associated momentum, which equal to the negative of system total energy leads to maximum increase in the gravitational field. The amount of energy that contributes to the maximum increase is $70.7 \%$ of the total energy. A Null Paraboliod is proposed and interpreted in terms on the space and momentum of time. Modeling of what is so-called gravity field waves, gravity carriers (the gravitons) is presented in this proposed paper. This model suggests that the space-time has a polarity and is composed of dipoles which are response- ble for forming the orbits and storing the space-time energy-momentum. The tri-dipoles can be unified into a solo space-time dipole with an angle of 45 degrees. Such a result shows that the space-time is not void, on the contrary, it is full of conserved and dynamic energymomentum structure. In this model the gravity field waves is assumed to be carried by the graviton and moves in the speed of light. The equivalent mass of the graviton (the lightest particle in nature up to the author's knowledge) is found to be equal to 0.707 of the equivalent mass of the light carrier (the photon) $\left(2.5119 \times 10^{-52}\right.$ $\mathrm{kg})$.

Considering a very infinitesimally period of time similar to that of the inflation $10^{-33}$ sec happened early in the universe. Calculations show that, our universe will experience an impulse force in the order $10^{83} \mathrm{~N}$. Because the impulse force will act in a direction opposite to gravitational force line of action, then, the gravitational bounding force will collapse, and so the universe will.

\section{REFERENCES}

[1] Einstein, A. (1997) The foundation of the general Theory of Relativity. In: English translation edited by A. J. Kox, M. J. Klien, and R. Schulmann, The Collected Papers of Einstein, 6, Princeton University Press, New Jersey, pp. 146-200.

[2] Knop, R.A., Aldering, G., Amanullah, R., et al. (2003) New constraints on $\Omega_{M}, \Omega_{\Lambda}$, and $\Omega_{\Lambda}$ from an independent set of eleven high-redshift supernovae observed with HST1. The Astrophysical Journal. http://sonic.net/ rknop/php/astronomy/papers/knop2003/ knopetal2003.pdf

[3] Permutter, S., et al. (1999) Measurements of omega and lambda from 42 high redshift supernovae. Astrophysical Journal, 516, 565-586.

[4] Riess, A.G., et al. (1998) Observational evidence from supernovae for an accelerating universe and cosmological constant. Astronomical Journal, 116, 1009-1038. doi:10.1086/300499

Maartens, R. and Majerotto, E. (2006) Observa- tional constraints on self-accelerating cosmology. Journal of Astrophysics. arXiv:astro-ph/0603353v4

[5] Perlmutter, S., Turner, M.S. and White, M. (1999) Constraining dark energy with $\mathrm{SNe}$ Ia and large-scale structure. Journal of Astrophysics.

arXiv:astro-ph/9901052v2

[6] Freedman, W.L. et al. (2000) Final results from the Hubble Space Telescope Key Project to measure the Hubble Constant. Journal of Astrophysics, 553, 47-72.

doi:10.1086/320638

Tonry, J.L., et al. (2003) Cosmological results from High-z Supernovae. Journal of Astrophysics, 594, 1-24. doi: $10.1086 / 376865$

[7] Carroll, S.M. Sawicki, I. Silvestri, A. and Trodden, M. (2006) Modified-source gravity and cosmological structure formation. Journal of Astrophysics, $\mathbf{8}$.

[8] Cengel, Y.A. and Boles, M.A. (2006) Thermodynamics: 
An engineering approach. 5th Edition, McGraw Hill, Columbus.

[9] Noether, E. (1918) Inavariante variationsprobeleme. Nachrichten von der Gesellschaft der Wissenschaften zu Goettingen, 235-257.

[10] Spergel, D.N., et al. (2006) Willkinson Microwave Anisotropy Probe (WMAP) three years results: implications for cosmology. NASA publications.

[11] Turner, M.S. (1999) Dark matter and dark energy in the universe. The Third Astronomy Symposium: The Galactic Halo ASP Conference Series, 666.

[12] Huterer, D. and Turner, M.S. (1999) Prospects for probing the dark energy via supernova distance measurements. Physical Review D, 60.

[13] Carroll, S.M., Hoffman, M. and Trodden, M. (2003) Can the dark energy equation-of-state parameter $\mathrm{w}$ be less than -1 ? Physical Review D, 68.

[14] Huterer, D. and Turner, M.S. (2001) Probing dark energy: Methods and strategies. Physical Review D, 64.

[15] Peebles, P.J.E. and Ratra, B. (2003) The cosmological constant and dark energy. Reviews of Modern Physics, 75, 559-606. doi:10.1103/RevModPhys.75.559

Copeland, E.J., Sami, M. and Tsujikawa, S. (2006) Dynamics of Dark Energy. International Journal of Modern Physics D, 15, 1753-1935. doi:10.1142/S021827180600942X

[16] Volovik, G.E. (2006) Vcauum energy: myths and reality. International Journal of Modern Physics A, 15, 19872010.

[17] Carroll, S.M. (2003) Why is the universe accelerating? Journal of Astrophysics.

[18] Carroll, S.M. (2006) The cosmological constant. Living Reviews of Relativity, 4.

[19] Shibli, M. (2007) The foundation of the Fourth Law of
Thermodynamics: Universe Dark Energy and its nature can Dark Energy be generated? To be presented at International Conference on Renewable Energies and Power Quality (ICREPQ'07), Spain.

[20] Jarosik, N., et al. (2007) Three-year Wilkinson Microwave Anisotropy Probe (WMAP) observations. Astrophysics Journal, 170, in Press.

[21] Dvali, G. and Turner, M. (2003) Dark energy as a modifications of the Friedmann Equation. Journal of Astrophysics.

[22] Shibli, M. (2006) Canonical modeling approach of a micro/nano free-flying space robot: a proposal towards detecting the nature of space-time. 1st IEEE ISSCAA, Co-sponsored by AIAA, Harbin, China.

[23] Shibli, M. (2006) Physical insight of universe Dark Energy: the space mission. 1st IEEE International Symposium on Systems and Control in Aerospace and Astronautics (ISSCAA 2006), Co-sponsored by AIAA, Harbin.

[24] Spergel, D.N., et al. (March 2006) Wilkinson Microwave Anisotropy Probe (WMAP) three year results: implications for cosmology. WMAP collaboration.

[25] Zhurkin, V.B. (1983) Specific alignment of nucleosomes on DNA correlates with periodic distribution of purinepyrimidine and pyrimidine-purine dimers. FEBS Letters, 158, 293-297. doi:10.1016/0014-5793(83)80598-5

[26] Goldstien, H. (1980) Classical mechanics, $2^{\text {nd }}$ Ed., Addison-Wesley, Boston.

[27] Shibli, M. (2006) Physical insight of space-time and modeling of space-time dipoles, gravity waves and gravitons: A micro space antenna to detect the nature of gravity waves. 1st IEEE International Conference on Advances in Space Technologies (ICAST2006), Pakistan.

[28] Lanczos, C. (1966) The variational principle of mechanics, University of Toronto Press, Toronto. 